\title{
Nonantibiotic prevention and management of recurrent urinary tract infection
}

Néha Sihra ${ }^{1}$, Anna Goodman², Rhana Zakri ${ }^{1}$, Arun Sahai ${ }^{1}$ and Sachin Malde ${ }^{*}$

Abstract | Urinary tract infections (UTIs) are highly prevalent, lead to considerable patient morbidity, incur large financial costs to health-care systems and are one of the most common reasons for antibiotic use worldwide. The growing problem of antimicrobial resistance means that the search for nonantibiotic alternatives for the treatment and prevention of UTI is of critical importance. Potential nonantibiotic measures and treatments for UTIs include behavioural changes, dietary supplementation (such as Chinese herbal medicines and cranberry products), NSAIDs, probiotics, D-mannose, methenamine hippurate, estrogens, intravesical glycosaminoglycans, immunostimulants, vaccines and inoculation with less-pathogenic bacteria. Some of the results of trials of these approaches are promising; however, high-level evidence is required before firm recommendations for their use can be made. A combination of these agents might provide the optimal treatment to reduce recurrent UTI, and trials in specific population groups are required.

Urinary tract infections (UTIs), which are defined microbiologically as the inflammatory response of the urothelium to microbial pathogens, are among the most common bacterial infections affecting an estimated 150 million people each year worldwide ${ }^{1}$. The true incidence is difficult to accurately define (owing to differences in reporting methods and diagnostic criteria), but half of all women have been estimated to experience a UTI in their lifetime, and up to $50 \%$ of these will have recurrent infection within the following 6-month period ${ }^{2,3}$. Observational studies have found an average recurrence rate in women of 2.6 infections per year ${ }^{4}$. The incidence of UTI in men is considerably lower than that in women, with an estimated lifetime prevalence of $13.7 \%$. In 2007 , UTI recurrence accounted for 10.5 million outpatient consultations and 2-3 million emergency department visits in the USA alone $^{5}$. In addition, UTIs are the most common cause of infection in hospitalized patients, accounting for $17.2 \%$ of all nosocomial infections in England ${ }^{6}$. Furthermore, UTIs result in considerable patient morbidity and time off work; hence, the management of this condition incurs large financial costs, estimated at $\$ 3.5$ billion in the USA per year ${ }^{7,8}$.

No definition of recurrent UTI is universally accepted, but it is commonly defined as at least two episodes of symptomatic infection (dysuria, frequency, urgency, suprapubic pain or haematuria), with pyuria or positive bacterial culture, in the past 6 months or three infections in the past 12 months. Recurrent UTI is associated with short-term morbidity, with a questionnaire-based study of college-aged women reporting an estimated 6.1 days of symptoms, 2.4 days of restricted activity and 1.2 days of time off work as a result of a UTI episode?.

UTIs are highly prevalent, account for large numbers of outpatient and emergency department consultations and are the leading cause of hospital-acquired infection and subsequent morbidity, thereby incurring large financial costs to health-care systems, highlighting the public health burden of this condition. However, more important than these factors is the growing risk of antimicrobial resistance as a result of our current method of managing these patients as $\sim 25 \%$ of all antibiotic prescriptions are for $\mathrm{UTIs}^{10}$. Antimicrobial resistance is now one of the most substantial threats to patient safety worldwide ${ }^{10}$. The European Centre for Disease Prevention and Control has estimated that antimicrobial resistance costs the European Union $\$ 1.5$ billion in health-care expenses and lost productivity each year ${ }^{11}$, and infection with antimicrobial-resistant bacteria can lead to more severe infections, longer hospital stays and increased mortality ${ }^{12}$.

This Review describes the pathogenesis of uncomplicated UTI and the need for nonantibiotic treatments and explores the nonantibiotic management options for the prevention and management of recurrent UTI in patients with a history of uncomplicated recurrent symptomatic UTI. The evidence for their use is described and 


\section{Key points}

- Rising rates of antimicrobial resistance, fuelled by the overuse of antibiotics in humans, are a serious threat to global public health.

- Alternatives to antibiotics for the prevention of recurrent urinary tract infection (UTI) are attractive options to reduce the risks of antimicrobial resistance.

- The most commonly studied nonantibiotic management options for recurrent UTI include cranberries, probiotics, D-mannose, methenamine hippurate, estrogens, intravesical glycosaminoglycans and immunostimulants.

- Studies of novel vaccines targeting the adherence mechanisms of uropathogenic bacteria seem promising, but human trials are required to determine the efficacy of this approach.

- Evidence for the nonantibiotic measures is hampered by considerable heterogeneity, and further placebo-controlled randomized trials of these agents are needed.

discussed, and gaps in our current knowledge and future directions for research are highlighted.

\section{Pathophysiology}

The pathogenesis of uncomplicated UTIs in women is thought to begin with contamination of the vaginal and periurethral areas with uropathogens that usually reside in the gut. In support of this theory, a study of vaginal introital cultures from 140 women found a significantly higher rate of vaginal Escherichia coli colonization in women with recurrent UTI than in those without $(35 \%$ versus $11 \% ; P<0.01)^{13}$. These vaginal $E$. coli isolates from women with recurrent UTI have also been shown to possess the same virulence factors as uropathogenic E. coli (UPEC) isolated from the urinary tract (such as $\mathrm{P}$ pili) ${ }^{14}$. Adherence of these uropathogens to vaginal epithelial and urothelial cells, migration to the bladder and colonization and internalization into urothelial cells with the subsequent formation of intracellular bacterial communities (IBCs) all depend on a complex host-pathogen interaction. Once internalized, bacteria can rapidly replicate within urothelial cells, forming communities that can evade host defence mechanisms, resulting in quiescent intracellular reservoirs ${ }^{15}$ (FIG. 1). In mouse models, these bacteria can lie dormant in the urothelial cells of the bladder for many months without being detected and could be a source of chronic, recurrent $\mathrm{UTI}^{15,16}$. Evidence in human trials is limited, but studies of urine cytology in women with recurrent UTI have shown the presence of IBCs ${ }^{17}$. The key molecular mechanisms involved in the formation of IBCs have been extensively studied ${ }^{7}$, and successful infection of the urinary tract is determined by the strength of bacterial virulence factors relative to the host's natural defence mechanisms.

\section{The need for nonantibiotic management}

The armamentarium of effective antibiotics is rapidly diminishing, and the size of this problem cannot be overstated $^{18}$. Resistance to amoxicillin is now $100 \%$ among urinary isolates of $E$. coli in some countries in Africa, and high levels of resistance to many commonly prescribed antibiotics have been identified worldwide ${ }^{19}$. Resistant strains of E. coli, such as ST131 (O25:H4), are associated with outbreaks of UTI, and the widespread emergence and spread of carbapenem-resistant Enterobacteriaceae (CRE) is a global public health threat ${ }^{18,20}$. Transmissible resistance in Enterobacteriaceae is now emerging against colistin (via $m c r-1$ ) with the potential to
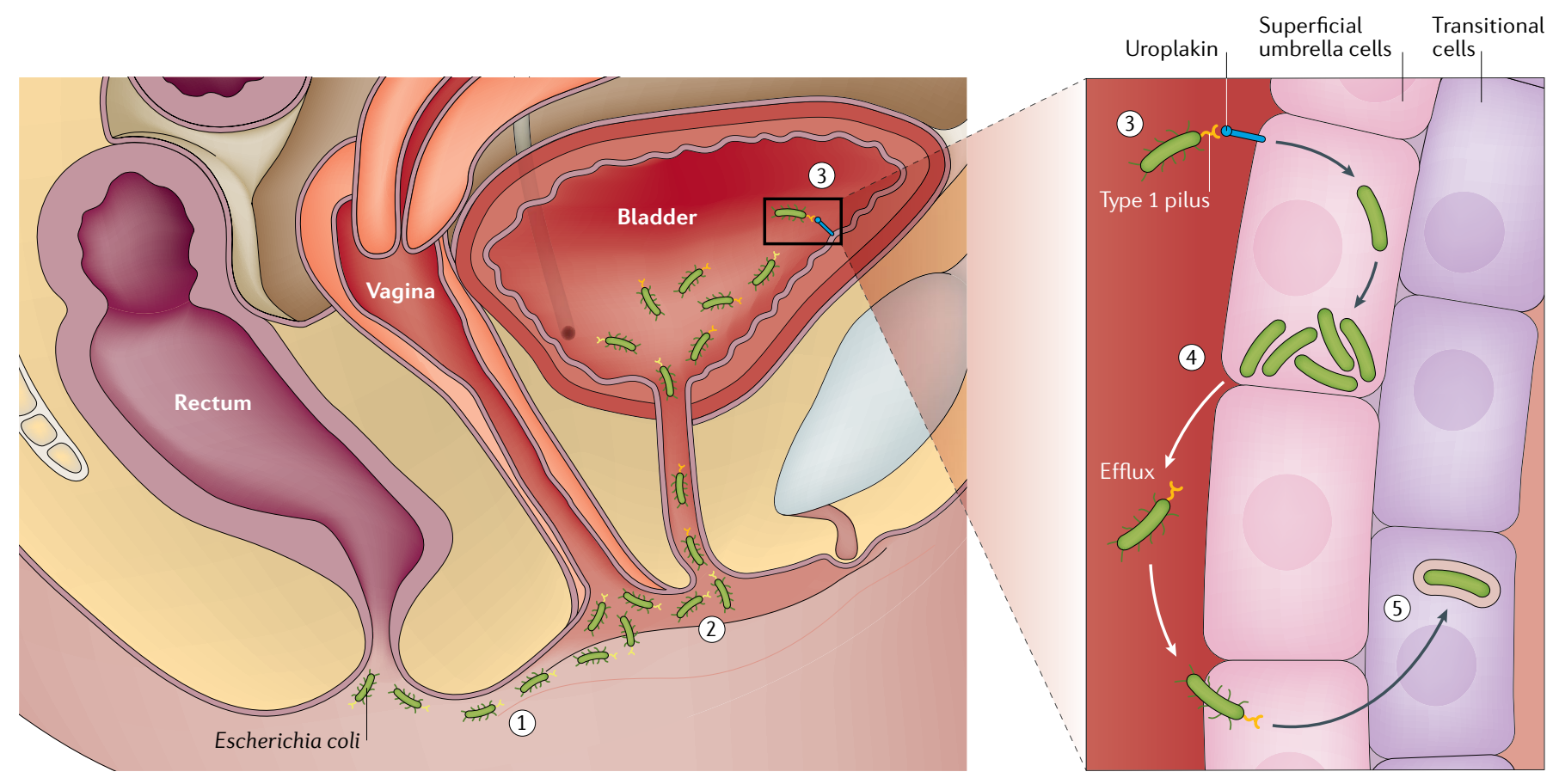

Fig. 1 | Pathogenesis of urinary tract infection. First, colonization of the vaginal and periurethral areas with uropathogens, such as uropathogenic Escherichia coli (UPEC), which usually reside in the gut, occurs (step 1). Second, these uropathogens migrate to the bladder (step 2). Third, UPEC type 1 pili adhere to uroplakins on superficial umbrella cells, leading to bacterial internalization (step 3). Fourth, UPEC multiply to form intracellular bacterial communities (IBCs) (step 4). Fifth, efflux of UPEC from IBCs results in reinvasion of neighbouring cells and the formation of quiescent intracellular reservoirs in transitional cells, which can remain viable for months (step 5). 

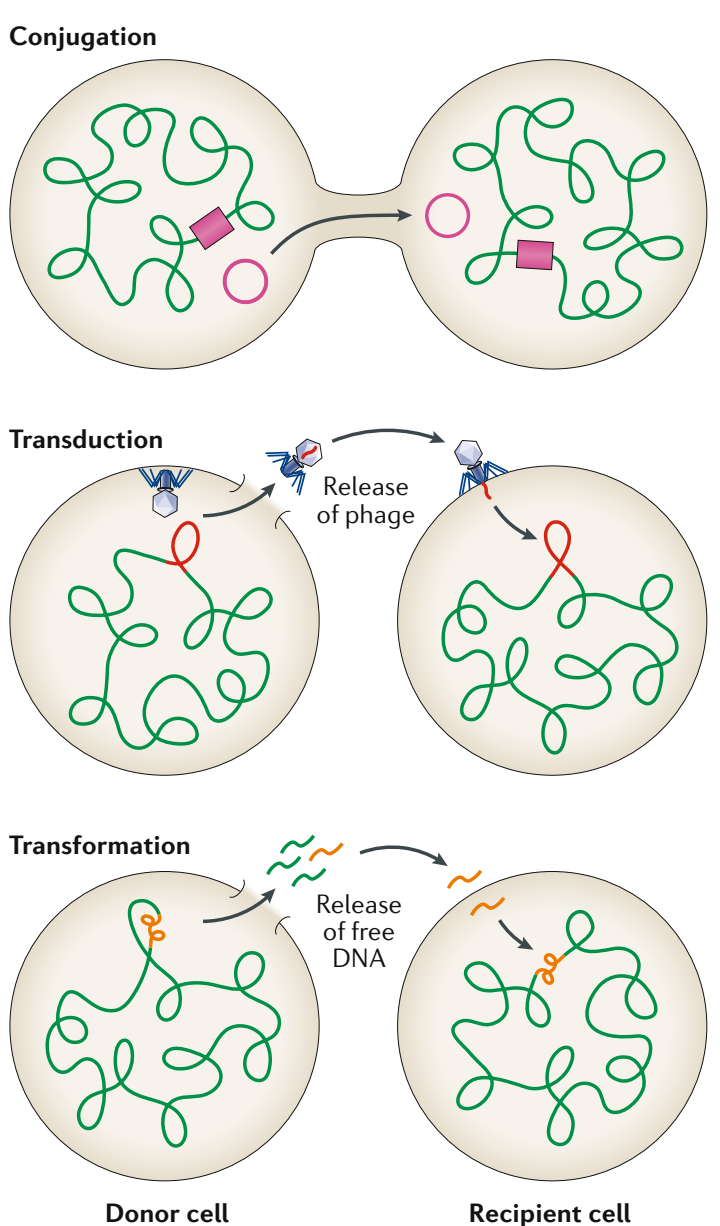

$$
\begin{aligned}
& \text { క్య Chromosome } \\
& \square \text { Transposon } \\
& \square \text { Plasmid }
\end{aligned}
$$

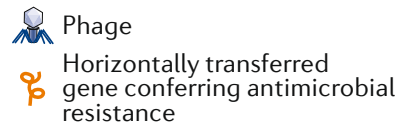

Fig. 2 | Mechanisms of antimicrobial resistance by mobile genetic elements. Mobile genetic elements such as plasmids and transposons enable rapid spread of resistance between bacteria. Such resistance through these mechanisms increases under antibiotic pressure via positive selection. Conjugation requires cell-to-cell contact to transfer DNA from one bacterial cell to another. In transduction, bacterial DNA is transferred from one cell to another by phages. In transformation, DNA released from one bacterial cell is taken up by another. Transduction and transformation do not require cell-to-cell contact.

rapidly spread ${ }^{21}$. This development means that our drug of last resort for treating infections caused by multidrugresistant Gram-negative bacteria is failing, and infection with these multidrug-resistant strains might, therefore, be untreatable with currently available antibiotics.

Rising antibiotic resistance, fuelled by increasing use of antibiotics in humans and animals, has occurred through the emergence of a combination of resistance mechanisms ${ }^{22,23}$. Resistance occurs owing to successive mutations, emerging chromosomal mutations, mobile genetic elements (MGEs) or a combination of these factors. MGEs, such as plasmids and transposons, enable rapid spread of resistance between bacteria
(FIG. 2). Such resistance through all of these mechanisms increases under antibiotic pressure via positive selection. Resistance mechanisms can act to inactivate an antibiotic, alter the concentration available at the point of action or modify the target of the antibiotic ${ }^{24}$.

Since the first $\beta$-lactamase (an enzyme that provides resistance to $\beta$-lactam-based antibiotics) was discovered in E. coli in 1940 (REF. $\left.{ }^{25}\right),>2,000$ similar enzymes have been discovered, including extended-spectrum $\beta$-lactamases (ESBLs), AmpC $\beta$-lactamases (AmpCs) and carbapenemases (such as KPC, NDM, IMP, VIM and OXA $)^{25,26}$. Combinations of $\beta$-lactam antibiotics with inhibitors of $\beta$-lactamase, such as piperacillintazobactam, can prevent inactivation of the antibiotic in some cases, but this defence is often eventually overcome, as bacteria evolve further resistance mechanisms. ESBLs are generally encoded by plasmids, which can be easily spread between organisms. The expression of AmpC genes is often caused by induction or derepression of chromosomal genes. Carbapenemases encoded on MGEs have rapidly spread between bacterial species, leading to major outbreaks of infection with CRE and increased mortality as the prevalence of CRE rises ${ }^{26}$. Alteration of the concentration of antibiotic reaching its target within the bacterium is controlled through efflux mechanisms and porin loss, and antibiotic pressure contributes to the induction of resistance mechanisms ${ }^{27}$. Porin loss in AmpC-producing and ESBLproducing Enterobacteriaceae can lead to carbapenem resistance in the absence of carbapenemases. However, this resistance has a fitness cost and might not spread as rapidly as MGE carbapenemases ${ }^{28}$. Efflux pump mutations, such as oqxAB, can confer resistance to nitrofurantoin in addition to nitrofuran reductase mutations. Modification of the antibiotic target is commonly seen in resistance to aminoglycosides and quinolones through alteration of the ribosome or DNA gyrase, respectively ${ }^{22}$ (TABLE 1).

Single resistance mechanisms occurring in isolation can usually be overcome by alternative antimicrobials. However, increasingly, Gram-negative pathogens with complex backgrounds exist, in which multiple mechanisms of resistance coexist in the same organism, leading to the emergence of multidrug-resistant uropathogens. Coexistence of resistance mechanisms can occur together on a single plasmid. For example, arm $A$, which confers resistance to aminoglycosides via ribosome methylation and alteration of the target site, has been found to be associated with the ESBL gene CTX-M in Klebsiella pneumoniae ${ }^{29}$. The prevalence of multidrugresistant $E$. coli identified in urinary samples from outpatients in the USA rose from $9 \%$ in 2001 to $17 \%$ in 2010 and continues to rise ${ }^{30}$. Predictors of resistance include prescription of the antibiotic within the previous 6 months and exposure to a geographical area with highly resistant organisms ${ }^{31}$.

We are facing a future in which combination therapy for UTI treatment will be routine, as resistance rates to single agents rise to unacceptable levels worldwide and untreatable UTIs present a real concern. This problem is exacerbated by the overuse of antibiotics, both in humans and in veterinary medicine. To control this crisis 
Table 1 | Mechanisms of resistance against commonly used antibiotics

\begin{tabular}{|c|c|}
\hline Antibiotic & Mechanism of resistance \\
\hline Aminoglycosides & $\begin{array}{l}\text { Decreased uptake of antibiotic by alteration of the } 30 \mathrm{~S} \\
\text { ribosomal subunit } \\
\text {-Antibiotic inactivation by production of aminoglycoside- } \\
\text { modifying enzymes }\end{array}$ \\
\hline$\beta$-Lactams & $\begin{array}{l}\text { - Modification of penicillin-binding proteins to prevent binding } \\
\text { of antibiotic } \\
\text {-Antibiotic inactivation by production of penicillinase }\end{array}$ \\
\hline Mannosides & - Decreased uptake of antibiotic by alteration of the $50 \mathrm{~S}$ subunit \\
\hline Quinolones & $\begin{array}{l}\text { - Reduced binding of quinolones by modification of DNA gyrase } \\
\text { and topoisomerase IV } \\
\text { - Efflux pumps exporting antibiotics out of the cell }\end{array}$ \\
\hline Nitrofurantoin & $\begin{array}{l}\text { - Efflux pumps exporting antibiotics out of the cell } \\
\text { - Mutations in nitrofuran reductase preventing antibiotic action }\end{array}$ \\
\hline Trimethoprim & $\begin{array}{l}\text { - Efflux pumps exporting antibiotics out of the cell } \\
\text { - Mutations in dihydrofolate reductase target enzyme } \\
\text { - Natural insensitivity }\end{array}$ \\
\hline
\end{tabular}

in antimicrobial resistance, nonantibiotic approaches are crucial in providing a means of reducing symptoms without resorting to antibiotic use.

Alternatives to antibiotics for the treatment, augmentation of treatment or prevention of recurrent UTI are attractive options to reduce the risks of antimicrobial resistance.

\section{Behavioural measures}

One of the key natural defence mechanisms against UTI is the presence of numerous different commensal organisms in the vaginal and periurethral areas, most commonly Lactobacillus species ${ }^{32}$. These commensal organisms are able to bind to vaginal epithelial cells (thereby preventing colonization of the vaginal and periurethral area by uropathogens) alter the local vaginal environment through changes in $\mathrm{pH}$ and release directly toxic chemicals (such as hydrogen peroxide), thereby reducing the potential for colonization by uropathogenic bacteria $^{33,34}$. Factors that affect normal vaginal microbiota can, therefore, increase the risk of developing UTIs.

Studies have shown that sexual intercourse within the past week is a risk factor for UTIs in premenopausal women, probably owing to increases in vaginal and bladder colonization with UPEC ${ }^{13,35-37}$. In a case-control study of almost 500 premenopausal women, those who experienced recurrent UTI ( $n=229$; defined as the presence of symptoms in association with microbiological confirmation in at least two of the episodes) were tenfold more likely to have had sexual intercourse more than nine times in the previous month, and twice as likely to have had a new sexual partner or used spermicide contraception in the previous year, than a control group of women who had no history of recurrent UTI $(n=253$; defined as no history of UTI in the previous 12 months and no history of more than one UTI in any 12-month period $)^{38}$. Similar findings were reported in a prospective observational study of 78 postmenopausal women who had a threefold to fourfold increased risk of symptomatic UTI 2 days after sexual intercourse ${ }^{39}$. However, another study of 316 healthy women aged $40-65$ years failed to show an increased risk of UTI after sexual intercourse, and risk factors for UTI could not be explained by menopausal status alone ${ }^{40}$. In this study, a number of other factors were shown to predispose postmenopausal women to UTI, including a history of UTI in the past year, urinary incontinence, antibiotic use in the previous 2 weeks and exposure to cold (defined by a positive answer to the question "In the past 2 weeks, have your hands, feet, back or buttocks been exposed to cold, and felt very cold to you for 30 minutes or more?"). In another case-control study of 149 postmenopausal women with a history of recurrent UTI compared with a control group of 53 women with no history of UTI, risk factors for UTI were incontinence, presence of elevated postvoid residual urine and cystocoele ${ }^{41}$. Thus, the risk factors for UTI in postmenopausal women are different from those seen in premenopausal women and are not linked to menopausal status alone. Anal intercourse would probably increase vaginal colonization with bowel flora, such as E. coli, increasing the risk of UTI, although this risk factor has not been well studied ${ }^{42}$. Careful discussion of sexual practices in the clinic is important, as avoidance of activity that is deemed to be a risk factor for UTI could prevent recurrence ${ }^{43}$.

The evidence for spermicide-based contraceptives (such as coated condoms, diaphragms and cervical caps) as a risk factor for recurrent UTI is strong ${ }^{35}$. The main active ingredient in spermicides (nonoxynol-9) is toxic to lactobacilli but not to $E$. coli, thereby potentially increasing vaginal colonization with potentially uropathogenic bacteria. In a study of premenopausal women, introital E. coli colonization was found to be significantly increased in 75 women using diaphragm-spermicide contraception (from $15 \%$ at baseline to $48 \%$ at week $1 ; P=0.001$ ), with no change in E. coli colonization in 103 women using oral contraceptives $^{44}$. The risk is highest for spermicide-coated condoms, but the use of condoms without spermicide has also been shown to increase the risk of developing UTIs. In a nested case-control study of sexually active premenopausal women with and without UTI, those exclusively using condoms coated with nonoxynol-9 $(n=23)$ had an OR for developing UTI of 2.2 (95\% CI 1.0-4.8), and those using exclusively nonspermicidal condoms $(n=8)$ had an OR of 1.6 (95\% CI 0.6-4.5) $)^{45}$. Thus, women presenting with recurrent UTI should be counselled about alternative methods of contraception.

A number of other behavioural factors are widely quoted in the lay press as increasing the risk of UTI (such as wiping back to front and douching). However, evidence is currently insufficient to quantify the risk that these behaviours pose. One case-control study based on a questionnaire survey did not find any statistically significant differences in voiding habits (such as delayed voiding, no voiding before or after intercourse or reduced daytime frequency) or personal hygiene practices (including wiping back to front, douching, tampon use or use of cotton underwear) between those with and those without recurrent UTI $(P>0.05)$, but a significant risk of recall bias was observed and, therefore, behavioural measures require further study in prospective trials ${ }^{38}$. In terms of voiding, a study of 366 women showed a significant reduction in the rate of UTI (from $9.8 \%$ to $1.6 \%$; $P<0.001$ ) following an educational intervention, which consisted of disseminating knowledge 
of UTI preventive strategies at new employee orientation, placing posters in the workplace and e-mail and oral communications. This reduction in UTI rate was associated with significant increases in water intake and urine voiding $(P<0.001)$, suggesting that voiding habit is linked to hydration and both might be protective in reducing UTI recurrence ${ }^{46}$.

On the basis of current evidence, sexual practices and use of spermicidal contraceptives are known risk factors for developing recurrent UTI, and patients should be counselled about the increased risk. Alternative contraceptive measures should be sought and behavioural measures such as voiding before and after intercourse should be discussed, although further evidence regarding voiding habits in this setting are required $^{47}$ (BOX 1).

\section{Dietary measures}

The role of simple dietary modification to prevent the development of UTI remains unproven, but the beststudied dietary measures are adequate hydration and the use of vitamin C (BOX 1).

\section{Hydration}

The role of hydration in preventing UTI has been the subject of a number of observational studies, but the majority of these investigations were published over 30 years ago. These studies are small, use various methodology and provide mixed results. Thus, confidently drawing any firm conclusions about the role of hydration in UTI prevention is not possible. Adatto and colleagues ${ }^{48}$ studied the fluid intake and voiding habits of 84 premenopausal women with a history of recurrent UTI and compared

\section{Box 1 | Evidence for nonantibiotic agents in preventing recurrent urinary tract infection in premenopausal women}

\section{Behavioural}

Recent sexual intercourse within the past week and use of spermicide contraceptives have been shown to increase the risk of developing urinary tract infection (UTI) in case-controlled and observational studies ${ }^{38}$. Evidence for voiding habit, douching and wiping back to front have not been well studied and require further assessment.

\section{Dietary}

Hydration and urinary concentration have been poorly studied as a way to reduce UTI recurrence. Evidence from survey-based studies has suggested that fluid restriction increases the risk of $\mathrm{UTI}^{49}$, but further study of fluid intake and modification of urinary $\mathrm{pH}$ and osmolality is required. Evidence for the role of ascorbic acid in UTI prevention is lacking.

\section{Phytotherapy}

\section{Chinese herbal medicine}

Studies of Chinese herbal medicine are small with a high risk of bias ${ }^{56}$. Further studies are required before this approach can be recommended for the prevention of recurrent UTI.

\section{Cranberry products}

Cranberry products might be effective in reducing recurrent UTI in women with a history of recurrent UTI. A Cochrane review has reported no statistically significant benefit for cranberry products ${ }^{85}$, but the level of heterogeneity among studies is high, and well-conducted, randomized, double-blind, placebo-controlled trials have reported positive results ${ }^{88,90}$, supported by a meta-analysis ${ }^{84}$. Further well-powered randomized trials with strict inclusion criteria and consistent dosing are required.

\section{NSAIDs}

Ibuprofen has been studied in two double-blind randomized controlled trials against antibiotics for the treatment of acute UTI with promising results ${ }^{107,108}$, but has not been studied as a prophylactic agent to prevent UTI recurrence.

\section{Probiotics}

A Cochrane review of seven randomized controlled trials has concluded that probiotics have no benefit over placebo in reducing UTI recurrence rates ${ }^{141}$. However, studies have high heterogeneity, and further large randomized trials using well-characterized Lactobacillus strains are warranted.

\section{D-Mannose}

D-Mannose has been studied as a prophylactic agent in one randomized controlled trial ${ }^{156}$, which reported positive results. This agent seems promising as a prophylactic agent in the prevention of recurrent UTI. However, this promise needs to be confirmed in larger, well-powered randomized trials.

\section{Methenamine hippurate}

A Cochrane review of 13 studies ${ }^{164}$ concluded that methenamine hippurate is associated with a reduction in the risk of recurrent UTI in patients with normal renal tracts. Only one of these randomized controlled trials evaluated methenamine hippurate as a prophylactic agent in the prevention of recurrent UTI, and the data are from small, poorly conducted trials. A large randomized trial is currently underway ${ }^{169}$.

\section{Estrogens}

The use of vaginal estrogens has been shown to reduce UTI recurrence rates in postmenopausal women in a Cochrane review of five randomized controlled trials - three against placebo and two against antibiotic prophylaxis $^{191}$.

\section{Intravesical glycosaminoglycans}

Two small randomized controlled trials of intravesical hyaluronic acid and chondroitin sulfate have reported positive results in reducing UTI recurrence rates ${ }^{211,212}$. Large well-powered studies are required to confirm these findings.

\section{Immunostimulants}

Uro-Vaxom

Uro-Vaxom (OM-89) has been found to reduce UTI recurrence in a metaanalysis of four randomized controlled trials ${ }^{96}$ but is effective only for Escherichia coli UTIs.

\section{Solco-Urovac}

Solco-Urovac has been shown to have a modest beneficial effect in UTI prevention in three randomized controlled phase II trials ${ }^{96}$, but larger randomized phase III studies are required before a conclusion can be reached regarding its efficacy.

Uromune

Uromune has shown excellent efficacy in retrospective cohort series ${ }^{232,233}$, but results from randomized placebo-controlled trials are awaited before recommendations on its use can be made.

\section{Vaccines}

Vaccines against various virulence factors of uropathogenic $E$. coli (such as fimbriae and toxins) have been studied in preclinical studies ${ }^{237-243}$, but only ExPEC4V has been investigated in a phase I trial in humans with promising results ${ }^{245}$. Studies in humans are required before these agents can be recommended for UTI prevention.

\section{Competitive inoculation}

The intravesical instillation of less-pathogenic $E$. coli into the bladder to prevent infection with more virulent strains has been studied with promising results in patients with indwelling catheters and incomplete bladder emptying ${ }^{246,247}$, but this method of UTI prevention requires further investigation in larger randomized controlled trials. 
the results with a control group of 84 women with no history of UTI. No difference in fluid intake or number of micturitions between the groups was observed. However, another study of 791 women with a mean age of 43 years (range 22-73 years) reported a 2.21-fold increased risk of UTI in the group with voluntary fluid restriction compared with those without ${ }^{49}$. However, important details were not studied, such as the mean volume of fluid intake in each group and the urinary osmolality. These types of omission limit the findings of the majority of studies in this area. Hydration and urinary osmolality are also poorly studied in relation to prophylaxis against recurrent UTI. A small study of 17 premenopausal women who experienced recurrent UTI (defined as two positive midstream urine (MSU) samples in the previous 6 months) showed that regular self-monitoring of urine osmolality resulted in improved hydration, reduced osmolality and fewer UTIs over a 4-month period, but further study is required to confirm these results ${ }^{50}$. Further research into the modification of urinary $\mathrm{pH}$ or osmolality as prophylaxis against UTIs is warranted.

\section{Ascorbic acid}

Ascorbic acid (vitamin C) was originally thought to prevent UTIs by acidification of the urine ${ }^{51}$. In a study of 12 hospitalized patients, $2.5 \mathrm{~g}$ of ascorbic acid in divided doses over 24 hours reduced mean urinary $\mathrm{pH}$ to 5.3 compared with a mean urinary $\mathrm{pH}$ of 7.4 in the placebo group ${ }^{52}$. However, in six patients with spinal cord injury (four men and two women), $500 \mathrm{mg}$ supplemental ascorbic acid four times a day had no significant effect on urinary $\mathrm{pH}(P>0.05)^{53}$. Studies of urinary acidification with ascorbic acid and its role in UTI prevention are limited by their small sample sizes, lack of control for confounding dietary factors that might alter urinary $\mathrm{pH}$ or lack of a placebo group. Hence, no conclusions can be drawn on the role of urinary acidification in the treatment or prevention of recurrent UTI. Thus, the preventive effect of ascorbic acid is unknown, and evidence for its use in this setting is lacking, but randomized trials with large numbers of participants examining the effect of ascorbic acid on urinary $\mathrm{pH}$ would help to determine the role of urinary acidification in the prevention of recurrent UTI (BOX 1).

\section{Phytotherapy}

The use of plants or plant components to treat medical conditions dates back centuries. In relation to the treatment and prevention of UTI, Chinese herbal medicine (CHM) and cranberry-containing products have been best studied.

\section{Chinese herbal medicine}

The plants used in CHM have been studied in randomized controlled trials both for the treatment of acute UTI and as prophylaxis to prevent recurrent UTI. In vitro, Chinese herbs, such as Huang Lian (Coptis chinensis Franch), have inhibitory activity against a number of uropathogenic bacteria (including E. coli) in addition to anti-inflammatory effects. Other herbs, such as Compound Salvia Plebeia Granules (CSPG), have been shown to reduce $E$. coli adherence to bladder urothelial cells. In a study of male rats, intragastric administration of $20-40 \mathrm{~g} / \mathrm{kg}$ of CSPG was found to have diuretic, antipyretic and analgesic effects, and it was also found to inhibit growth of $E$. coli with a minimal inhibitory concentration of $0.25 \mathrm{~g} / \mathrm{ml}\left(\mathrm{REF}^{54}\right)$. An in vitro study evaluated the antimicrobial properties of three different berberine alkaloids (the major active component of C. chinensis Franch) and found that the growth rate constant of $E$. coli was reduced with all berberine alkaloids studied (berberine, coptisine and palmatine) ${ }^{55}$. In studies of women with recurrent UTI, the most commonly studied CHM are Er Xian Tang, Bai Tou Weng Tang and San Jin Wan. A Cochrane review of seven randomized controlled trials with a total of 542 women evaluated the role of CHM in the treatment and prevention of recurrent $\mathrm{UTI}^{56}$. Three studies compared $\mathrm{CHM}$ with antibiotic treatments ${ }^{57-59}$, two compared CHM in combination with antibiotics with antibiotics alone $e^{60,61}$ and two compared two different CHM regimes ${ }^{62,63}$. The studies comparing CHM with antibiotic prophylaxis found that those receiving $\mathrm{CHM}$ had a considerably lower rate of recurrent UTI (relative risk (RR) 0.28 , 95\% CI $0.09-0.82)^{56}$. The addition of CHM to antibiotic prophylaxis was also shown to be more effective in reducing recurrent UTI rates than antibiotic prophylaxis alone (RR $0.53,95 \%$ CI $0.35-0.80)^{56}$. However, a number of substantial limitations must be taken into account when interpreting these findings. Studies were heterogeneous, included small numbers of patients and had a high risk of bias. All studied were classified as very low quality on the basis of the GRADE Quality of Evidence criteria ${ }^{64}$, and the risk of bias was high predominantly owing to inadequate blinding of participants and personnel as well as unclear descriptions of allocation concealment; blinding of outcome assessment; and selective reporting. Furthermore, five of the seven studies evaluated postmenopausal women ${ }^{57-60,62}$; thus, the evidence in the premenopausal population is limited. For these reasons, the use of CHM cannot be recommended until larger, higher-quality, well-conducted randomized trials are undertaken (BOX 1).

\section{Cranberry}

Evidence for the use of cranberries and cranberry-based products for the prophylaxis of UTIs remains mixed, and little evidence exists for its role in the treatment of an acute UTI. Cranberry is a plant from the family Ericaceae and is known as Vaccinium macrocarpon, Vaccinium oxycoccos and Vaccinium erythrocarpum. The berries are composed predominantly of water (88\%), followed by a complex mixture of organic acids, fructose, ascorbic acid, flavonoids, anthocyanidins, proanthocyanidins, catechins and triterpenoids ${ }^{65,66}$. Anthocyanidins and proanthocyanidins are tannins (polyphenols) that function as a natural plant defence system against microbial infection and are the components thought to be most clinically relevant in preventing UTIs in women ${ }^{67}$.

Initially used during the 17 th century for various therapeutic indications including gastric and hepatic disorders, scurvy and cancer, cranberry has been a popular therapy for UTIs for almost 100 years, particularly before the advent of antibiotics ${ }^{68,69}$. 
Proposed mechanism of action. To date, no definitive mechanism of action has been elucidated to explain the prophylactic properties of cranberries in preventing recurrent UTI. Pure cranberry juice is very acidic, with a $\mathrm{pH}$ of $<2.5\left(\mathrm{REF}^{65}\right)$. On the basis of this acidity, acidification of urine was initially hypothesized to contribute to the antibacterial effect of cranberry ${ }^{68}$. However, this hypothesis was rejected $>20$ years ago when in vitro studies established the importance of one of its key components - proanthocyanidins ${ }^{70}$. Adherence to urothelial cells is a critical event in the initiation of UTIs caused by $E$. coli, and this process is facilitated by adhesins (namely, type 1 fimbriae and $\mathrm{P}$ fimbriae (pyelonephritis fimbriae)), which enable bacteria to bind to the carbohydrate receptors on the surface of urothelial cells ${ }^{69}$. Almost all uropathogenic strains of E. coli express type 1 fimbriae, which bind to mannose-like receptors ${ }^{71}$. Strains of $E$. coli with increased virulence, isolated from patients with recurrent UTI and/or pyelonephritis, possess other variants of fimbria, notably $P$ fimbriae ${ }^{67}$. Cranberries contain fructose, which has been found to have a role in inhibiting the adherence of $E$. coli with type 1 fimbriae to urothelial cell receptors in vitro, and proanthocyanidins, which affect adherence of P fimbriated E. coli ${ }^{72}$ (FIG. 3). The presence of proanthocyanidins is thought to be the most important factor in mediating the clinical effect of cranberries in UTI prevention owing to their inhibitory effect on the motility of Pseudomonas aeruginosa, E. coli and Proteus mirabilis, as well as their antiadherence effect ${ }^{73,74}$. Proanthocyanidins are composed of oligomers and polymers of flavans. These oligomers and polymers are most often linked by a single bond (B-type), but a unique structural feature of proanthocyanidins in cranberries is the presence of a second bond (A-type linkage), and this A-type linkage has been predominantly implicated in the inhibitory effect of cranberry on $E$. coli adherence. This implication is based on a study assessing the bacterial antiadhesion activity of other proanthocyanidin-containing foods (such as grape juice, apple juice, green tea and dark chocolate) compared with

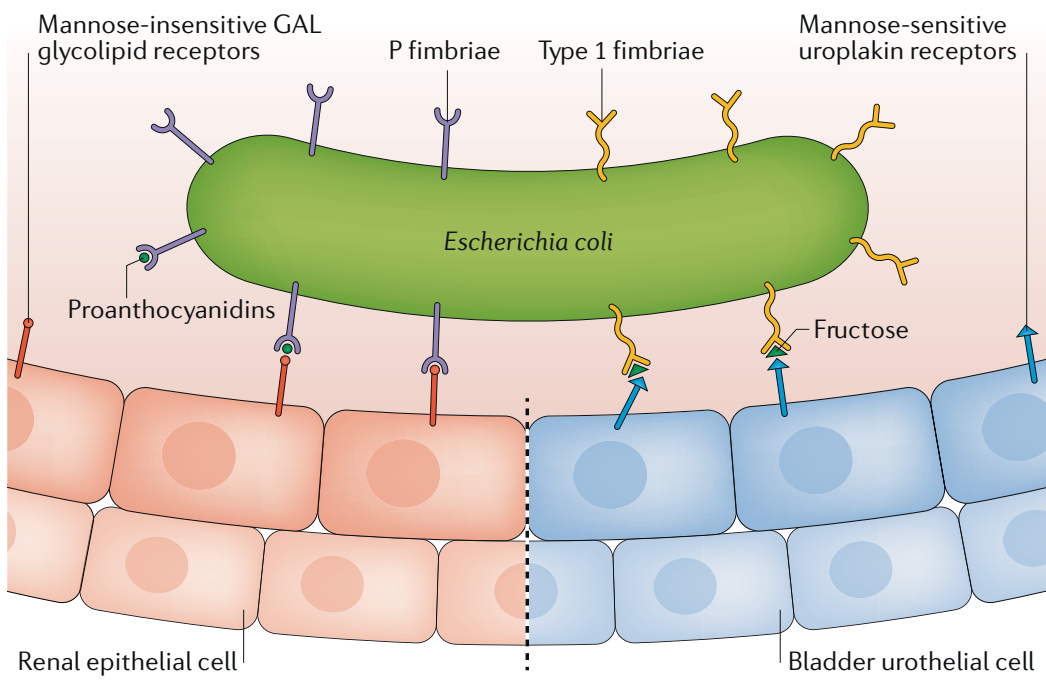

Fig. 3 | Mechanisms of action of cranberry components in prevention of urinary tract infection. Fructose inhibits the binding of type 1 fimbriae to uroplakin receptors. Proanthocyanidins prevent binding of $\mathrm{P}$ fimbriae to glycolipid receptors. that of proanthocyanidin from cranberry ${ }^{75}$. In this study, the urine from human volunteers who had consumed cranberry juice demonstrated increased antiadhesion activity compared with those who had consumed other proanthocyanidin-containing foods, and this effect was confirmed in vitro ${ }^{75}$. The primary difference was the presence of A-type linkages in the proanthocyanidins from cranberry juice compared with B-type linkages in those from grape juice, apple juice, green tea and dark chocolate. This observation suggests that the A-type linkage is responsible for the inhibitory effect of cranberries on $E$. coli adherence; this inhibitory effect might be caused by the fact that A-type linkages are similar in structure to the bacterial-binding receptors on urothelial cells and, therefore, these proanthocyanidins bind to bacterial fimbriae, preventing adherence of the bacteria to the urothelial cell ${ }^{75}$ (FIG. 4).

The precise mechanism through which cranberry components exert an antiadhesion effect remains unclear. Cranberry compounds might act as receptor analogues, competitively inhibiting E. coli adhesion to the urothelium by binding to the fimbrial tips ${ }^{67}$. Cranberry components might alter the cell surface properties of bacteria (such as a reduction in fimbrial length and density), thereby decreasing adherence to urothelial cells. Prolonged exposure to cranberry can result in morphological changes to E. coli from rod shaped to spherical, resulting in increased repulsion by urothelial cells ${ }^{76}$.

Vasileiou and colleagues ${ }^{77}$ suggested that other cranberry components (such as ursolic acid, a pentacyclic triterpenoid) have a complementary or synergistic role to the proanthocyanidin polyphenols in the antiadhesion process by causing differential gene expression in E. coli, resulting in inhibition of biofilm formation ${ }^{78}$. An in vitro study evaluated the effect of the plant extract ursolic acid on E. coli biofilm formation. Using 96-well plates and a flow chamber, $10 \mu \mathrm{g}$ of ursolic acid was found to inhibit E. coli biofilm formation by 6-20-fold more than controls ${ }^{78}$. DNA microarray analysis was used to study the gene expression profiles of $E$. coli in response to ursolic acid, and genes involved in chemotaxis and mobility (cheA, tap, tar and $\operatorname{mot} A B$ ) and heat shock response ( $h s l S T V$ and mopAB) were shown to be consistently induced, suggesting that increased motility prevents the formation of a mature biofilm ${ }^{78}$.

Mode of ingestion. Cranberry can be administered in several forms, including fresh whole berries, tablets, juice cocktails and extracts, and the concentration of proanthocyanidins varies between preparations. However, as expected, the complex mixture of bioactive components is found only in whole cranberry fruit ${ }^{79}$. The amount of cranberry juice cocktail required to prevent adhesion of UPEC to the urothelium has been suggested to be $240 \mathrm{ml}$. This suggestion is based on an in vitro study of 39 UPEC isolates from the urine of premenopausal women with culture-confirmed UTI, showing that after consumption of $240 \mathrm{ml}$ of cranberry juice cocktail, urine prevented adhesion of $80 \%$ of $E$. coli isolates, and the effect lasted up to 10 hours after ingestion $^{80}$. A randomized controlled trial comparing a daily intake of $300 \mathrm{ml}$ of a commercially available cranberry 


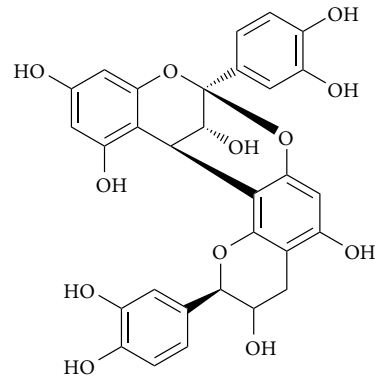

A-type linkage

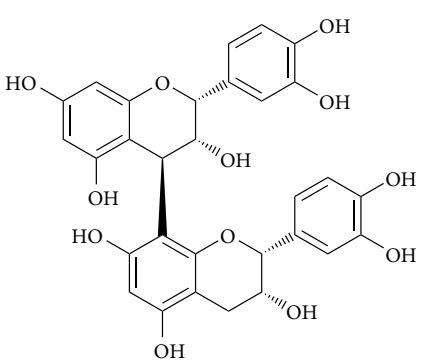

B-type linkage
Fig. 4 | A-type and B-type linkages of proanthocyanidins. The A-type linkage is probably responsible for the inhibitory effect of cranberries on Escherichia coli adherence; this inhibitory effect might be caused by the fact that A-type linkages are similar in structure to the bacterial-binding receptors on urothelial cells and, therefore, these proanthocyanidins bind to bacterial fimbriae, preventing adherence of the bacteria to the urothelial cell ${ }^{75}$. two microbiologically confirmed symptomatic UTIs in the past year aged 21-72 years, were included. Women were randomized to receive placebo juice plus placebo tablets $(n=50)$, placebo juice plus cranberry tablets $(n=50)$ or cranberry juice plus placebo tablets $(n=50)$. Tablets were taken twice a day, and $250 \mathrm{ml}$ of juice was taken three times a day. A significant reduction was observed ( $>50 \%$ decrease) in the proportion of women experiencing symptomatic UTI during the 12-month follow-up period in the cranberry juice and cranberry tablet groups compared with placebo - to $20 \%$ with cranberry juice and to $18 \%$ with cranberry tablets compared with $32 \%$ with placebo $(P<0.05)$. The mean number of UTIs in the year after treatment was 0.72 in the placebo group, 0.30 in the cranberry juice group $(P<0.05)$ and 0.39 in the cranberry tablet group $(P<0.05)$. No significant difference in the rate of reported UTIs was observed when comparing the cranberry juice and cranberry tablet groups ${ }^{87}$. The overall risk of bias was judged by the investigators to be low in this study, but the dose of proanthocyanidins was not specified, and the investigation was not adequately powered to detect a meaningful difference between the treatment and control groups.

In another double-blind, randomized, placebocontrolled trial, Vostalova and colleagues ${ }^{88}$ compared the use of cranberry fruit powder (with a clearly defined proanthocyanidins dosage) with placebo. Overall, 182 women aged $18-75$ years with a history of two symptomatic UTIs in the previous year were included, and 83 were randomized to receive $500 \mathrm{mg}$ of cranberry fruit powder (containing $2.8 \mathrm{mg}$ of proanthocyanidins) per day or to receive placebo. During the 6-month followup period, the proportion of women having more than one UTI was significantly lower in the cranberry group (10.8\%) than in the placebo group (25.81\%) $(P=0.04)$. These findings corresponded to a RR reduction of $58 \%$ in the cranberry group compared with placebo, and this study had a low risk of bias ${ }^{88}$. Maki and colleagues ${ }^{89}$ reported the results of a 24 -week, multicentre, doubleblind, randomized, controlled trial comparing $240 \mathrm{ml}$ of cranberry juice per day $(n=185)$ with placebo $(n=188)$ in women with a history of recurrent UTI. Recurrent UTI was defined as two or more episodes of selfreported UTI without microbiological confirmation. A $39 \%$ reduction in clinical UTI episodes with cranberry juice and a $37 \%$ reduction in clinical UTI with pyuria episodes were observed. No difference in the rate of microbiologically confirmed UTIs was observed, and overall one UTI was prevented for every 3.2 personyears $^{89}$. Kontiokari and colleagues ${ }^{90}$ compared the effect of cranberry-lingonberry juice or Lactobacillus GG drink with placebo in 150 women with a history of one UTI caused by E. coli ( $>10^{5}$ colony-forming units (CFU)/ml E. coli). Patients were treated with antibiotics for the acute infection and had subsequent confirmation of a negative urine culture before being randomized 1:1:1 to receive cranberry-lingonberry juice, Lactobacillus GG drink or placebo. Patients were monitored for 12 months, and cranberry-lingonberry juice resulted in a $20 \%$ reduction in the absolute risk of recurrence compared with placebo $(P=0.023)$, whereas 
no difference in risk of recurrence was reported between the Lactobacillus GG drink and the placebo group. The study was intended to run over 12 months, but patients in the cranberry-lingonberry juice group were monitored for only 6 months as the manufacturer stopped producing this juice. During this 6-month period, 8 women (16\%) in the cranberry-lingonberry group, 19 (39\%) in the Lactobacillus GG drink group and $18(36 \%)$ in the control group had at least one episode of UTI. However, the nonblinded nature of this trial is a limitation and source of potential bias, and this study reported outcomes specifically in patients with UTIs caused only by E. coli.

Trial results on the effect of cranberry on UTI have not all been positive. One study compared the use of two different doses of cranberry juice ( $4 \mathrm{oz}$ or $8 \mathrm{oz}$ daily) with placebo in 176 premenopausal women with a history of recurrent UTI (defined as one or more clinically diagnosed UTI in the previous year). No difference in the number of clinically confirmed or cultureconfirmed UTIs or difference in time to first UTI was reported between the cranberry and placebo groups at the 6-month follow-up point $(27.5 \%$ and $30.4 \%$, respectively; $P=0.70)^{91}$. However, a trend towards a protective effect of cranberry juice was observed, with the proportion of women with P fimbriated $E$. coli isolates considerably reduced in both cranberry groups $(43.5 \%$ in the cranberry groups and $80 \%$ in the placebo group, $P=0.07)$. These findings suggest that a cranberry-related protective effect does occur, and the authors suggested that larger, well-powered studies are needed to determine whether cranberry is effective in reducing UTIs ${ }^{91}$. A large double-blind, randomized, placebo-controlled trial compared the use of cranberry juice $(8 \mathrm{oz}$ twice daily) with placebo juice (same colour and taste as the cranberry juice, but without any cranberry component) in 319 women with a mean age of 21 years. Patients were included following one episode of acute uncomplicated UTI; thus, participants were not a specific group of patients with recurrent UTI. Furthermore, all patients were asked to drink $240 \mathrm{ml}$ of fluid twice a day (whether in the intervention or control group) as part of the study protocol. No significant difference in reduction of UTIs was observed ${ }^{92}$; however, the overall recurrence rate in both groups was much lower than would be expected from the literature (16.9\% compared with an expected $30 \%{ }^{93}$ ). A possible reason for this discrepancy was that the placebo juice contained ascorbic acid, and this component might have had a role in preventing UTI recurrence in the placebo group, thereby confounding the outcome. Furthermore, the protocol of the study might have led to improved hydration in the placebo group, possibly reducing UTI recurrence and confounding the primary outcome.

Clinical evidence in comparison with antibiotic prophylaxis. Two studies have compared the efficacy of cranberry extract with that of low-dose antibiotics. McMurdo and co-workers found cranberry juice capsules to be almost as effective as prophylactic trimethoprim in reducing the risk of recurrent UTI in a randomized study involving 137 women aged
$>45$ years $^{94}$. Women with recurrent UTI (defined as two clinically diagnosed UTIs in the previous 12 months) were randomized to receive $500 \mathrm{mg}$ cranberry extract daily or trimethoprim $100 \mathrm{mg}$ once daily and were monitored for 6 months. The difference in risk of developing a UTI between the two groups was not statistically significant, with fewer adverse effects in the cranberry group. However, a study in younger women (median age 34.8 in the cranberry group and 36.1 in the antibiotic group) compared the use of cranberry capsules with trimethoprim-sulfamethoxazole in a double-blind, randomized trial ${ }^{95}$. Overall, 221 premenopausal women with recurrent UTI (defined as three episodes of selfreported UTI in the previous 12 months) were given either $480 \mathrm{mg}$ trimethoprim-sulfamethoxazole daily or $500 \mathrm{mg}$ cranberry capsules twice daily and were monitored for 12 months. Antibiotic prophylaxis was more efficacious in reducing the risk of recurrent UTI, but at the expense of increasing antibiotic resistance ${ }^{95}$.

A Cochrane review by Jepson and colleagues ${ }^{85}$ published in 2012 included 24 studies totalling 4,473 patients. The risk reduction of recurrent UTI with cranberry treatment was not statistically significant (RR 0.86 , 95\% CI 0.71-1.04), and no benefit was found for any of the following subgroups when the data from all studies were pooled: women with recurrent UTI, older people, pregnant women, children with recurrent UTI, cancer patients or people with neurogenic bladder ${ }^{85}$. However, studies were heterogeneous with regard to the definition of UTI used, the patient population studied and the formulation of cranberry investigated, and most studies did not report the concentration of the active ingredient used. A number of other systematic reviews have reported positive results. Wang and co-workers ${ }^{86}$ performed a meta-analysis of 10 randomized trials including 1,494 patients. Cranberry-containing products were found to be effective in reducing the number of UTI in certain cohorts: women with recurrent UTI, women in general and children. However, considerable heterogeneity between studies (with regard to the population studied, the definition of UTI used and the cranberry product investigated) limits the conclusions reached. Beerepoot and Geerlings ${ }^{96}$ performed a systematic review and meta-analysis of two randomized placebo-controlled trials only in women with recurrent UTI (more than three UTIs in the past year). Results showed that cranberry products reduced UTI recurrence in this specific group (RR 0.53, 95\% CI 0.33-0.83). Finally, a systematic review and meta-analysis of 28 studies including 4,947 patients showed that the use of cranberry products significantly reduced (risk ratio 0.6750 , 95\% CI $0.5516-0.7965 ; P<0.0001)$ the risk of UTIs, especially in patients with a history of recurrent UTI (although the definition of recurrent UTI in this review was not described ${ }^{84}$. However, these findings should be confirmed in larger, well-powered trials before definitive conclusions can be made.

Specific population groups. The role of cranberry in reducing UTI recurrence has been studied in a number of specific populations, including patients with neurogenic bladder disorders, elderly individuals (aged $>65$ years), 
children and pregnant women. Patients aged $>65$ years in long-term care facilities were studied by Caljouw and colleagues ${ }^{97}$. Cranberry capsules were effective in reducing the number of clinically diagnosed UTIs (on the basis of the presence of specific and nonspecific micturitionrelated symptoms or signs or a positive urine dipstick test for nitrites or leukocytes) in patients with a high risk of developing a UTI (those with indwelling catheters for $>1$ month, a history of diabetes mellitus and/or more than one UTI in the past 12 months) (62.8 versus 84.8 per 100 person-years at risk; $P=0.04$ ), but no difference was observed between the cranberry and placebo groups in patients without these risk factors ( 40.3 versus 33.4 per 100 person-years at risk; $P=0.30$ ). In the same study, using a stricter definition of UTI recurrence by including a MSU growth of $>10^{5} \mathrm{CFU} / \mathrm{ml}$ to define UTI in addition to the clinical criteria described above, no statistically significant beneficial effect of cranberry over placebo was observed (treatment effect $1.02(95 \%$ CI 0.68-1.55; $P=0.91))^{97}$. Another study of 185 elderly nursing home residents with a mean age of 86 years reported no significant improvement in rates of bacteriuria or pyuria with cranberry capsules (containing $72 \mathrm{mg}$ of proanthocyanidins per day) compared with placebo at 1 year $(29.1 \%$ versus $29.0 \%$; OR $1.01,95 \%$ CI $0.61-1.66 ; P=0.984)^{98}$. However, the study population was fairly small, and the majority of patients were not suffering from recurrent UTI.

The role of cranberry in children and pregnant women has also been studied. A study performed in toilet-trained children up to age 18 years with a history of two or more previous UTIs, in the absence of anatomical urological abnormalities, showed a $65 \%$ reduction of UTIs with the use of cranberry at 1 year (incidence of UTI 0.4 per patient per year compared with 1.15 in the placebo group; $P=0.045)^{99}$. Another randomized trial of 84 girls aged 3-14 years found that consumption of $50 \mathrm{ml}$ of cranberry juice daily resulted in a significant reduction in symptomatic UTIs compared with $100 \mathrm{ml}$ of Lactobacillus GG drink administered for 5 days a month or placebo at the 6-month follow-up point (incidence of UTI: $18.5 \%$ in the cranberry group, $42.3 \%$ in the Lactobacillus group and $48.1 \%$ in placebo group; $P<0.05)^{100}$. In a study of 188 pregnant women $<16$ weeks into pregnancy, women were randomly allocated to one of three groups: cranberry juice cocktail three times a day $(n=58)$; cranberry juice at breakfast, then placebo at lunch and dinner $(n=67)$; or placebo three times a day $(n=63)$. The rates of symptomatic UTI were reduced by $41 \%$ in the group taking cranberry three times a day, but the study was not adequately powered to detect a significant difference in this outcome measure, and the dropout rate owing to gastrointestinal upset was high $(39 \%)^{101}$. In terms of safety, a systematic review by Dugoua and colleagues ${ }^{102}$ found no concerns to mother or fetus with the antenatal consumption of cranberry fruit juice, although its safety and harm during breastfeeding is unknown.

In summary, evidence is mixed on the use of cranberry and cranberry-containing products as prophylaxis for recurrent UTI (BOX 1). The majority of studies have small numbers of participants and different formulations and doses of cranberry. However, several well-conducted randomized controlled trials suggest that cranberry products might be efficacious in specific populations (women with recurrent UTI, women in general and children). Cranberry products are easily accessible, have a low adverse-effect profile and do not carry the same risk of bacterial resistance as antibiotics. Women with recurrent UTI should be informed of the mixed evidence of efficacy, but low adverse-effect profile, if they wish to use cranberry for the prevention of UTIs. However, for strong recommendations to be made, larger, well-designed trials are required.

\section{Nonsteroidal anti-inflammatories}

Early host-pathogen interactions and the severity of the host's acute inflammatory response within the first 24 hours following infection are likely to determine the risk of developing chronic cystitis (persistent symptoms, as opposed to the complete resolution of symptoms that characterizes acute UTI), as has been demonstrated in mouse models ${ }^{103}$. The presence of severe pyuria, bladder mucosal injury and elevated levels of specific cytokines (IL-5, IL-6, IL-8 and G-CSF) are predictors of the development of chronic cystitis ${ }^{103}$. Interestingly, suppression of this inflammatory response by preadministration of dexamethasone (a corticosteroid) before induction of UTI reduced the risk of developing chronic cystitis in mice $(2 \text { of } 17 \text { versus } 13 \text { of } 18 ; P<0.001)^{103}$. The chronic bladder inflammation that occurs as a result of long-term exposure to bacteria results in lymphonodular hyperplasia and $\mathrm{B}$ cell infiltration in the bladder submucosa and has been seen in studies of patients with neurogenic bladder and recurrent $\mathrm{UTI}^{104}$. Levels of serum cytokines and growth factors involved in monocyte and macrophage development were found to be increased in women with recurrent UTI compared with those without ${ }^{105}$. Furthermore, cyclooxygenase 2 (Cox2) mRNA expression is increased in mouse models of UTI by up to 50 -fold in the 24 hours following E. coli infection, and COX2 expression is increased in urine particulates from patients with $\mathrm{UTI}^{105,106}$. Inhibition of COX2 in a mouse model led to a significant reduction in the severity of pyuria after UTI, with reduced bacterial titres in the bladder and a decreased incidence of recurrent chronic cystitis (31\% (8 of 26) versus $77 \%$ (20 of 26$) ; P<0.05)^{105}$. These results suggest a possible beneficial role for NSAIDs or COX2 inhibitors in the treatment of acute UTI and in preventing the development of recurrent UTI.

\section{Clinical evidence}

No studies evaluating NSAIDs as a prophylactic agent to prevent UTI recurrence have been conducted (BOX 1). Two randomized controlled trials have evaluated the use of the NSAID ibuprofen for the treatment of acute uncomplicated UTIs in women. Bleidorn and colleagues ${ }^{107}$ performed a double-blind trial comparing ibuprofen with the antibiotic ciprofloxacin, both administered for 3 days, in 79 women presenting with an acute UTI. On day 4, 58\% of patients in the ibuprofen group were free of symptoms compared with $52 \%$ in the ciprofloxacin group $(P=0.744)$. However, $33 \%$ of patients in the ibuprofen group required secondary 
antibiotic treatment for persistent symptoms, compared with $18 \%$ in the ciprofloxacin group, and negative urine cultures at day 7 were higher in the ciprofloxacin group, but this was not statistically significantly $(71.9 \%$ versus $48.5 \%$; $P=0.093$ ). The main limitation of this study was the fact that it was not sufficiently powered to determine superiority of ibuprofen over ciprofloxacin. Another double-blind study compared ibuprofen with the broad-spectrum antibiotic fosfomycin in 494 women with an acute uncomplicated UTI ${ }^{108}$. The requirement for antibiotics by day 28 after UTI was significantly lower in the ibuprofen group (35\% versus $100 \% ; P<0.0001$ ), and $67 \%$ of women in the ibuprofen group recovered without antibiotic treatment. However, women receiving ibuprofen had a higher burden of symptoms at day 7 and a higher incidence of developing pyelonephritis than women who received antibiotic. Furthermore, previous studies have shown that the natural history of UTI is one of spontaneous resolution without treatment in $28 \%$ of patients ${ }^{109}$, and another randomized trial comparing the antibiotic nitrofurantoin with placebo in women with acute UTI reported that $54 \%$ of patients in the placebo arm had symptomatic improvement at day 3 , and $40 \%$ had a negative MSU at day 7 (REF. ${ }^{110}$ ). Thus, results regarding the effectiveness of NSAIDs for UTI must be interpreted with caution but warrant further investigation in large randomized trials. Interestingly, the COX2 inhibitor celecoxib has been shown to reverse multidrug resistance and increase the susceptibility of bacteria to common antibiotics ${ }^{111}$. In this in vitro study, celecoxib was not bactericidal but was found to increase the sensitivity of bacteria to the antibiotics ampicillin, kanamycin, chloramphenicol and ciprofloxacin. Using an ethidium

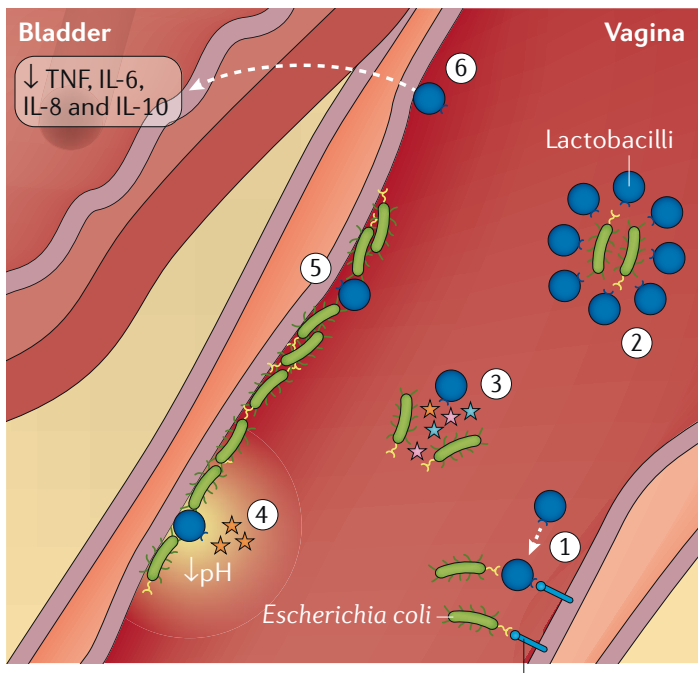

Vaginal epithelial receptors

Fig. 5 | Mechanisms of action of lactobacilli in urinary tract infection prevention. Lactobacilli can prevent urinary tract infection through many mechanisms, including competitive inhibition of uropathogen binding to vaginal epithelial cells (step 1); congregation around uropathogens (step 2); production of bacteriocins, hydrogen peroxide and lactic acid (step 3); acidic environment caused by lactic acid (step 4); inhibition of bacterial biofilm formation (step 5); and downregulation of pro-inflammatory cytokines (step 6). TNF, tumour necrosis factor. bromide efflux assay, the inhibitory effect of celecoxib was shown to be caused by blockage of the bacterial multidrug resistance efflux pump, thereby increasing antibiotic accumulation in bacterial cells. These findings suggest a novel intervention for treating UTIs caused by drug-resistant bacteria. This agent requires further study in trials of treatment of UTI in women.

\section{Probiotics}

Probiotics are live microorganisms that are thought to confer health benefits when consumed. The normal bacterial flora in humans is thought to be a key defence mechanism against infection; thus, augmenting this normal flora with probiotics is an attractive strategy to prevent infection.

\section{Mechanism of action}

In healthy women, the vagina is a balanced ecosystem in which normal flora have a critical role in protecting it against colonization by pathogenic organisms that can cause UTIs. Lactobacillus species are the predominant commensal organisms present in the vaginal and periurethral areas and are responsible for preventing adherence and migration of pathogens to the bladder urothelium $^{112}$. A significant reduction in vaginal lactobacilli levels has been shown to be associated with an increased rate of vaginal $E$. coli colonization (OR 4.0; $P=0.01)^{13}$, and a depletion in levels also occurs after sexual intercourse, spermicide use and in postmenopausal women, all of which have been shown to be risk factors for UTIs ${ }^{13,32,113}$. Repeated antibiotic use can also adversely affect the local lactobacilli composition of the vagina, and in a study of 70 women treated with antibiotics for UTI, a vaginal swab 4-6 hours after treatment showed lactobacilli colonization in only $14 \%$ of women ${ }^{114}$.

Lactobacilli are thought to exert their protective effects through many mechanisms (FIG. 5). These organisms compete with uropathogens for vaginal epithelial adhesion receptors, thereby preventing uropathogenic organisms from colonizing the vagina ${ }^{115}$. Adhesion can be blocked by exclusion (occupying binding sites so that uropathogens cannot initially bind), competition (directly competing with uropathogens for available adhesion receptors on vaginal epithelial cells) or displacement (displacing uropathogens that have already bound to vaginal epithelial cells). Coaggregation of lactobacilli with uropathogens, thereby creating a microenvironment in which the antimicrobial substances produced by lactobacilli (such as hydrogen peroxide, lactic acid and bacteriocin) are concentrated near the uropathogens, results in inhibition of bacterial biofilm formation and downregulation of proinflammatory cytokines (such as tumour necrosis factor, IL-6, IL-8 and IL-10) ${ }^{116-119}$. An in vitro study examined the antiadherence effects of three Lactobacillus strains (Lactobacillus salivarius UCM572, Lactobacillus acidophilus 01 and Lactobacillus plantarum CLC17) on uropathogenic bacteria (E. coli ATCCR53503, E. coli 10791, Enterococcus faecalis 04-1, E. faecalis 08-1 and Staphylococcus epidermidis 08-3) to an in vitro model of bladder epithelial cells (T24) ${ }^{120}$. Certain Lactobacillus strains (L. salivarius UCM572 and L. acidophilus 01) 
were found to prevent the adhesion of common uropathogens to the bladder urothelium, thereby indicating a role beyond vaginal colonization ${ }^{120}$. Furthermore, lactobacilli have been shown to have antimicrobial effects against ESBL-producing E. coli as well as multidrugresistant organisms, with the largest effects seen for L. plantarum and Lactobacillus fermentum strains ${ }^{121,122}$. In an in vitro study, 11 Lactobacillus isolates were screened against multidrug-resistant uropathogens (Candida albicans, K. pneumoniae, $P$. aeruginosa, E. coli and E. faecalis), and all Lactobacillus isolates were found to have a growth inhibition zone of $>10 \mathrm{~mm}$ against all the uropathogenic test organisms, with L. plantarum and L. fermentum showing the largest effects with growth inhibition zones of up to $28 \mathrm{~mm}^{121}$. Another in vitro study of six Lactobacillus strains (Lactobacillus gasseri HLAB 414, Lactobacillus rhamnosus HY7801, L. acidophilus SNUL, L. plantarum AK8-4, Lactobacillus paracasei $\mathrm{CH} 15-2$ and L. acidophilus Antibio300 screened against four uropathogens (ESBL(-) E. coli, ESBL(+) E. coli, Proteus vulgaris and E. faecalis) showed similar findings, with an average inhibitory zone size between 10.5 and $20.5 \mathrm{~mm}$ for Lactobacillus species, compared with $<6.0-27.5 \mathrm{~mm}$ for antibiotics, depending upon the resistance patterns of the uropathogens ${ }^{122}$. Overall, the antibacterial activity of lactobacilli was less potent than the sensitive antibiotics but more potent than the resistant antibiotics $(P<0.05)$. However, these in vitro findings need to be confirmed in vivo to understand the potential clinical benefits of different lactobacilli strains in the prevention and treatment of UTIs.

Competition for nutrients, production of substances that are directly toxic to uropathogenic bacteria (such as hydrogen peroxide, bacteriocins and bacteriocinlike substances) and creation of an acidic vaginal $\mathrm{pH}$ (through production of lactic acid) are other mechanisms by which lactobacilli have been shown to reduce vaginal and urethral colonization with uropathogens ${ }^{112,123}$. One study has shown that the toxic metabolites produced by lactobacilli (hydrogen peroxide and lactic acid) act cooperatively to kill uropathogenic organisms in vitro, with hydrogen peroxide demonstrating enhanced killing activity in the presence of lactic acid ${ }^{124}$. A proposed hypothesis to explain this finding is that lactic acid increases the permeability of the Gram-negative outer cell membrane to antimicrobial compounds (such as hydrogen peroxide), thereby increasing their bactericidal effects; using a fluorescent probe uptake assay, lactic acid has been shown to increase the permeability of the cell membrane of E. coli in vitro ${ }^{125}$. Lactobacilli have also been shown to have a role in modulating the host immune system. Lactobacilli can activate the Toll-like receptor (TLR) pathway, producing interleukins and myeloid differentiation factor 88 (MYD88), thereby mounting an immune response against uropathogens ${ }^{126}$. L. rhamnosus GR-1 has been shown to specifically potentiate the nuclear factor $-\kappa B$ $(\mathrm{NF}-\kappa \mathrm{B})$ pathway (which is activated by UPEC) by releasing various immunomodulatory proteins (such as NLP/P60, GroEL and elongation factor Tu ${ }^{127}$. These immunomodulatory proteins might be novel targets for future nonantibiotic drug development.
The relative contribution of all these different antimicrobial properties to overall clinical efficacy is unknown, as not all Lactobacillus strains possess all of these properties. An in vitro study assessing 15 different Lactobacillus strains found that Lactobacillus crispatus had a greater ability to block uropathogen adherence to vaginal epithelial cells than the other lactobacilli studied, whereas other strains (such as Lactobacillus jensenii) had a greater ability to directly inhibit growth of uropathogens ${ }^{116}$. Another study found that only $82 \%$ of all Lactobacillus strains studied produced hydrogen peroxide, and only $68 \%$ produced bacteriocin ${ }^{123}$. Considerable differences exist between strains, and these differences are important when assessing their potential role as therapeutic agents to prevent UTIs.

\section{Clinical evidence}

Despite the theoretical benefits of restoring levels of normal lactobacilli commensals in patients with recurrent UTI, clinical trials have reported mixed results. This lack of consistency is caused by the large heterogeneity in terms of strain of Lactobacillus used, mode of delivery, dosage and length of treatment. Furthermore, outcomes might be affected by the type of population studied (for example, women of different races might have different normal vaginal microbiome compositions $^{128}$ ). Thus, different strains might produce different clinical outcomes dependent upon many variables. The route of delivery, whether vaginal or oral, might affect the level of colonization of lactobacilli in the vagina. Importantly, clinically significant benefits have been reported only for well-characterized Lactobacillus strains irrespective of route of administration; thus, clinical trials of lactobacilli compounds should report the use of well-characterized strains. A study of 47 women using a non-characterized L. acidophilus strain that did not colonize the vagina showed no difference in reducing UTI rates ${ }^{129}$. Furthermore, in healthy postmenopausal women, daily oral administration of yoghurt containing $10^{9} \mathrm{CFU}$ of L. rhamnosus GG for a month did not lead to any significant increase in vaginal colonization ${ }^{130}$. However, using well-characterized strains, L. rhamnosus GR-1 and Lactobacillus reuteri RC- 14 could be detected in the vagina of a 33-year-old woman with a history of recurrent UTI for $>3$ weeks after the insertion of a single vaginal pessary containing L. rhamnosus GR-1, and the patient had no further UTI during the 7-week study period ${ }^{131}$. Oral administration has also been shown to result in vaginal colonization after transit through the gastrointestinal tract, although the number of lactobacilli that can reach the vagina in this way is lower than direct vaginal instillation and depends on the viability of the strains as they pass through the gut ${ }^{132,133}$. However, an advantage of the oral route could be the possible additional effect of lactobacilli in reducing the transfer of uropathogenic gut organisms from the rectum to the vagina ${ }^{133}$. Mezzasalma and colleagues ${ }^{134}$ have shown that oral administration of antimicrobial multispecies probiotic formulations (containing L. acidophilus and L. reuteri, or L. plantarum, L. rhamnosus and Bifidobacterium animalis subsp. lactis) leads to their increased presence in 
the vaginas of 40 premenopausal women when administered daily for 14 days, which can reduce uropathogenic bacteria and yeast numbers ${ }^{135}$. A randomized placebo-controlled trial of 64 healthy women with no previous history of UTI showed that daily oral administration of capsules of L. rhamnosus GR-1 and L. reuteri $\mathrm{RC}-14$ given together led to a significant increase in vaginal lactobacilli numbers at day $28(\log 10$ increase compared with placebo; $P=0.08)$ and $60(0.4 \log 10$ increase compared with placebo; $P=0.05$ ) compared with the control group and can convert some women from a state of having bacterial vaginosis into not having this disorder ${ }^{135}$.

Oral probiotics. Four randomized controlled trials have been conducted on the effects of oral probiotics as a prophylactic agent in the prevention of recurrent UTI, with mixed results ${ }^{90,100,136,137}$. Two studies randomly assigned patients to a $100 \mathrm{ml}$ L. rhamnosus GG drink, $50 \mathrm{ml}$ of cranberry juice or control (no intervention) ${ }^{90,100}$. Ferrara and colleagues ${ }^{100}$ evaluated 84 girls (mean age 7.5 years, range $3-14$ years) with more than one E. colipositive UTI in the previous year. L. rhamnosus GG was used at a dose of $4 \times 10^{7} \mathrm{CFU}$ and was taken for 5 days a month for 6 months. Cranberry juice contained $7.5 \mathrm{~g}$ of cranberry concentrate and $1.7 \mathrm{~g}$ of lingonberry concentrate in $50 \mathrm{ml}$ of water without sugar additives and was taken daily for 6 months. The incidence of symptomatic UTI was significantly reduced in the cranberry juice arm compared with the Lactobacillus group ( $18.5 \%$ versus $42.3 \%$; $P<0.05$ ), but no difference was seen between the Lactobacillus and control arms. In a similar study, 150 adult women with recurrent UTI (defined as more than one E. coli UTI in the previous year) were randomized to $100 \mathrm{ml}$ L. rhamnosus GG for 5 days a month for 1 year (at a dose of $4 \times 10^{10} \mathrm{CFU}$ ), $50 \mathrm{ml}$ cranberry-lingonberry juice daily for 6 months or control (no intervention) ${ }^{90}$. At 6 months, a symptomatic recurrent UTI was reported in $16 \%$ of participants in the cranberry-lingonberry group, $39 \%$ in the Lactobacillus group and $36 \%$ in the placebo group, again suggesting that Lactobacillus had no benefit in reducing recurrence of UTI. However, this result might have been caused by the dosing regimen used or the inability of this strain of Lactobacillus to colonize the vagina. The lack of effect seen in these studies should not, therefore, be generalized to all Lactobacillus strains. In support of this recommendation, a study of 120 patients (aged 13-36 years) with recurrent UTI (defined as a bacterial growth of $>10^{3} \mathrm{CFU} / \mathrm{ml}$ in suprapubic aspirated urine culture or $>10^{5} \mathrm{CFU} / \mathrm{ml}$ in catheterized urine culture (nontoilettrained children) and clean-caught urine culture (toilet-trained children)) and primary vesicoureteric reflux randomized to oral $L$. acidophilus or trimethoprim-sulfamethoxazole antibiotic prophylaxis for 1 year showed that use of Lactobacillus was equivalent to antibiotic prophylaxis in reducing UTI recurrence ${ }^{137}$. Another study of 85 boys and girls (aged 3-15 years) comparing nitrofurantoin antibiotic prophylaxis alone with L. acidophilus and B. animalis subsp. lactis plus nitrofurantoin antibiotic prophylaxis found that the combination was more effective in reducing the incidence of febrile UTI than prophylactic antibiotics alone $e^{138}$. Fewer studies of oral probiotics in adult populations with recurrent UTI have been conducted than those conducted in children. A randomized trial of 252 postmenopausal women with recurrent UTI (three episodes of self-reported UTI in the previous 12 months) randomized to oral L. rhamnosus GR-1 and L. reuteri RC-14 or antibiotic prophylaxis with trimethoprim showed that probiotics reduced the rate of recurrent UTI from 6.8 to 3.3 at 1 year (but the study criteria for noninferiority of $10 \%$ against antibiotic prophylaxis were not met). However, no antibiotic resistance occurred in the probiotic group, whereas the rate of resistance in the antibiotic group was as high as $95 \%$ after 1 month of treatment ${ }^{136}$; thus, oral probiotics could be a superior option to antibiotics for many patients.

Vaginal probiotics. Use of vaginal Lactobacillus has mostly been studied in adults. In one of the earliest randomized controlled trials, Baerheim and colleagues ${ }^{129}$ randomly allocated 47 women with recurrent UTI (defined as one or more episode of MSU-proven infection) to an intravaginal suppository containing $\geq 7.5 \times 10^{8} \mathrm{CFU}$ of $L$. casei var. rhamnosus twice a week for 26 weeks or placebo. No significant difference in the recurrence rate of UTIs (defined as symptoms in addition to $>1.0 \times 10^{4} \mathrm{CFU} / \mathrm{ml}$ or any quantity of Staphylococcus saprophyticus) was found between the two groups. However, this observation was not surprising as no difference was observed in the rate of periurethral colonization with this non-characterized Lactobacillus between the groups. Using two wellcharacterized strains, L. casei var. rhamnosus GR-1 and L. fermentum B-54, Reid and colleagues ${ }^{139}$ randomly allocated 41 adult premenopausal women (mean age 23 years) who had just been treated for an acute UTI to a vaginal suppository containing $1.6 \times 10^{9}$ organisms per capsule twice a week for 2 weeks, then once a month for 2 months $(n=19)$, or to a sterilized skimmed milk placebo capsule in the same regime $(n=21)$. UTI recurrence was reduced at 6 months in the treatment group compared with the placebo group (21\% compared with $47 \%$, risk ratio 0.46 (95\% CI 0.15-1.40)). In another study, 100 adult premenopausal women (aged 18-40 years, median age 21 years) with a history of recurrent UTI (at least one symptomatic UTI in the previous year) were randomly assigned on a 1:1 basis to a vaginal suppository containing $1 \times 10^{8} \mathrm{CFU} / \mathrm{ml}$ of $L$. crispatus once daily for 5 days then weekly for 10 weeks or placebo ${ }^{140}$. At 10 weeks, a considerable reduction was observed in the number of UTIs in the Lactobacillus group compared with the placebo group (15\% versus $27 \%$; RR $0.5,95 \%$ CI $0.2-1.2$ ). Furthermore, the increased vaginal colonization with L. crispatus correlated with reduced risk of UTI during the follow-up period.

A 2015 Cochrane review of all randomized controlled trials or quasi-randomized controlled trials comparing probiotics with no therapy, placebo or other prophylactic interventions (such as antibiotics) concluded that vaginal probiotics provided no significant benefit over placebo $^{141}$ (BOX 1). However, significant heterogeneity among the trials was noted, data were derived from 
small studies and methodological reporting was poor in many. Another meta-analysis suggested that probiotics were safe and effective in preventing recurrent UTI, but a definitive recommendation could not be made owing to the small numbers of patients and studies involved ${ }^{142}$. The use of well-characterized strains of Lactobacillus seems promising, and larger trials are required in order to enable firm conclusions to be made regarding their use. An understanding that not all probiotic strains are the same is important, and clinical efficacy needs to be demonstrated for each particular strain that is being recommended to patients.

\section{D-Mannose \\ Mechanism of action}

$\mathrm{D}$-Mannose is a monosaccharide isomer of glucose and is involved in the glycosylation of certain proteins (for example, monoclonal antibodies). Absorption of D-Mannose is rapid when administered orally, and it is detectable in the plasma $\sim 30$ minutes after consumption and ultimately is excreted in the urinary tract $^{143}$. Virulence factors of enteric bacteria, such as E. coli, include type 1 fimbriae or pili, which are proteinaceous appendages extending from the bacterial cell and are integral for attachment ${ }^{144}$. The type 1 pili are composed of Fim proteins with a FimH adhesion molecule at the tip of the fibrillum. The FimH adhesion lectin domain enables it to bind to mannosylated host proteins (the terminal mannose units of uroplakin 1a, Tamm-Horsfall glycoprotein and $\beta 1$ and $\alpha 3$ integrins) on the surface of the urinary tract ${ }^{145-148}$ (FIG. 6). Mouse models of UTI caused by E. coli have shown FimH to be critical in its pathogenesis; thus, FimH would be a good therapeutic target for future treatments ${ }^{149}$. As D-mannose is similar in structure to the binding site of urothelial glycoprotein receptors (such as uroplakin), in sufficient concentration in urine, D-mannose can cause saturation of FimH adhesins and prevent bacteria from binding to urothelial glycoprotein receptors ${ }^{150}$ (FIG. 7). In rats inoculated with E. coli by intravesical injection, a preparation containing $10 \% \mathrm{D}$-mannose was shown to reduce bacteriuria compared with control treatment (normal saline) at days 1 and $5(P<0.05$ and $P<0.01$, respectively $)^{150}$. Furthermore, an in vitro study evaluated the effect of D-mannose on the adherence of 73 different $E$. coli strains, which had been isolated from the urine, vagina or perianal area of women with recurrent UTI ( $90 \%$ of which demonstrated adherence to vaginal and buccal epithelial cells in vitro $)^{151}$. D-Mannose was found to completely inhibit the adherence of $42 \%$ of these E. coli strains to vaginal epithelial cells and inhibited another $18 \%$ of strains by at least $50 \%{ }^{151}$.

Synthetic mannosides have been developed that have a much greater affinity for the FimH ligand than $\mathrm{D}$-mannose, with in vitro adhesion of $E$. coli to the human bladder cell line 5637 being completely inhibited by a 100 -fold lower concentration of the synthetic mannoside heptyl- $\alpha$-D-mannose than $D$-mannose ${ }^{152}$. Heptyl-a-D-mannose has an optimized alkyl length for interaction with FimH (FIG. 8), and, once bound, the bacteria are irreversibly inhibited in their ability to bind to the urothelial surface, resulting in reduced invasion
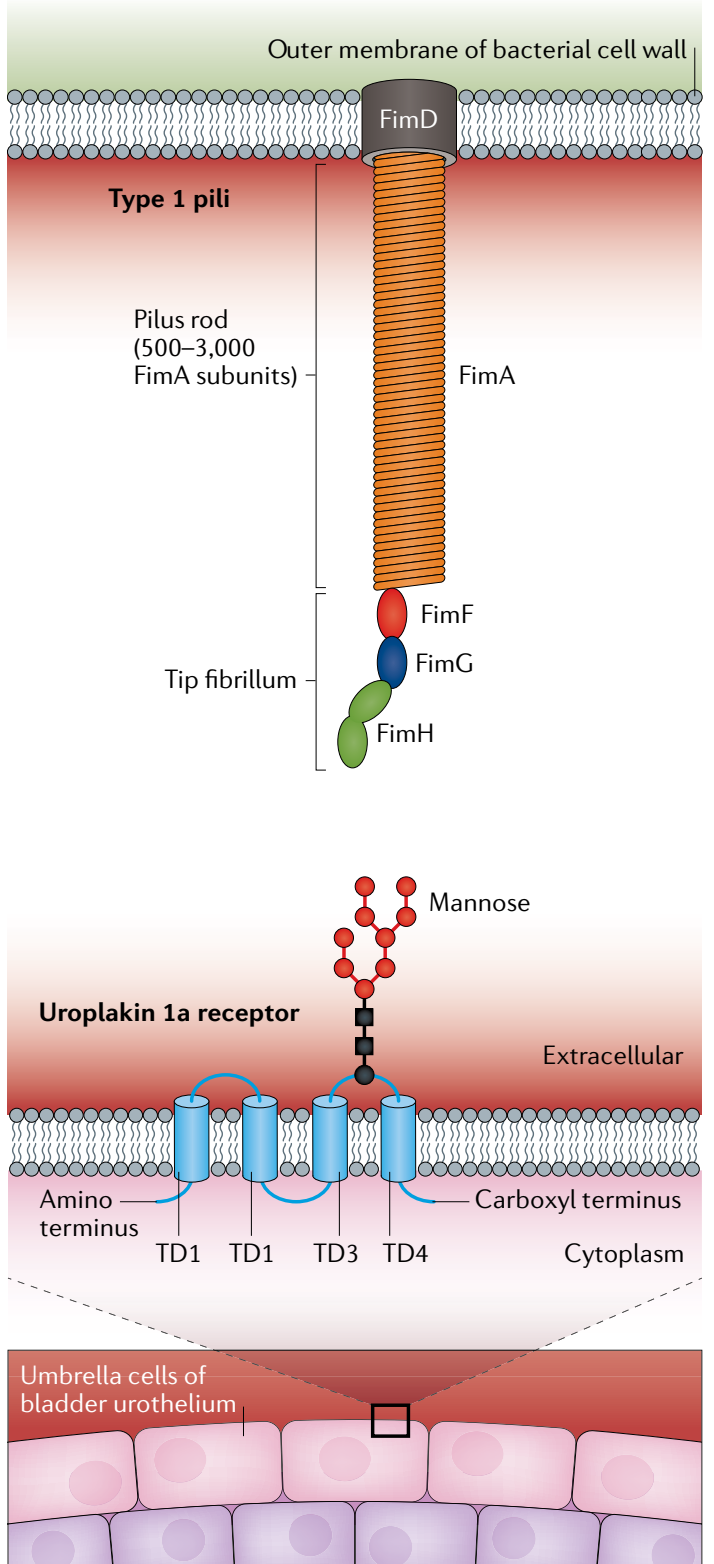

Fig. 6 | Type 1 pili of Escherichia coli and the uroplakin 1 a receptor. The type I pili are composed of Fim proteins with a FimH adhesion molecule at the tip of the fibrillum. The FimH adhesion lectin domain enables it to bind to mannosylated host proteins on the surface of the urinary tract. TD, transmembrane domain.

and IBC formation ${ }^{152}$. Studies in mice have shown that these synthetic FimH antagonists have a role in UTI prophylaxis as well as in treating acute UTI. Mice given a FimH antagonist by intraperitoneal injection or orally 30 minutes before being infected with UPEC administration to the bladder were found to have a significantly lower bacterial load at 6 hours following administration of the FimH antagonist compared with control mice treated with phosphate-buffered saline $(P<0.001)$; similar findings were reported in mouse models of chronic cystitis $^{153}$. Characterization of the X-ray crystal struc-

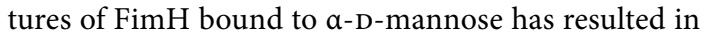
the development of synthetic biphenyl mannosides, such as M4284, which has an 100,000-fold higher binding 


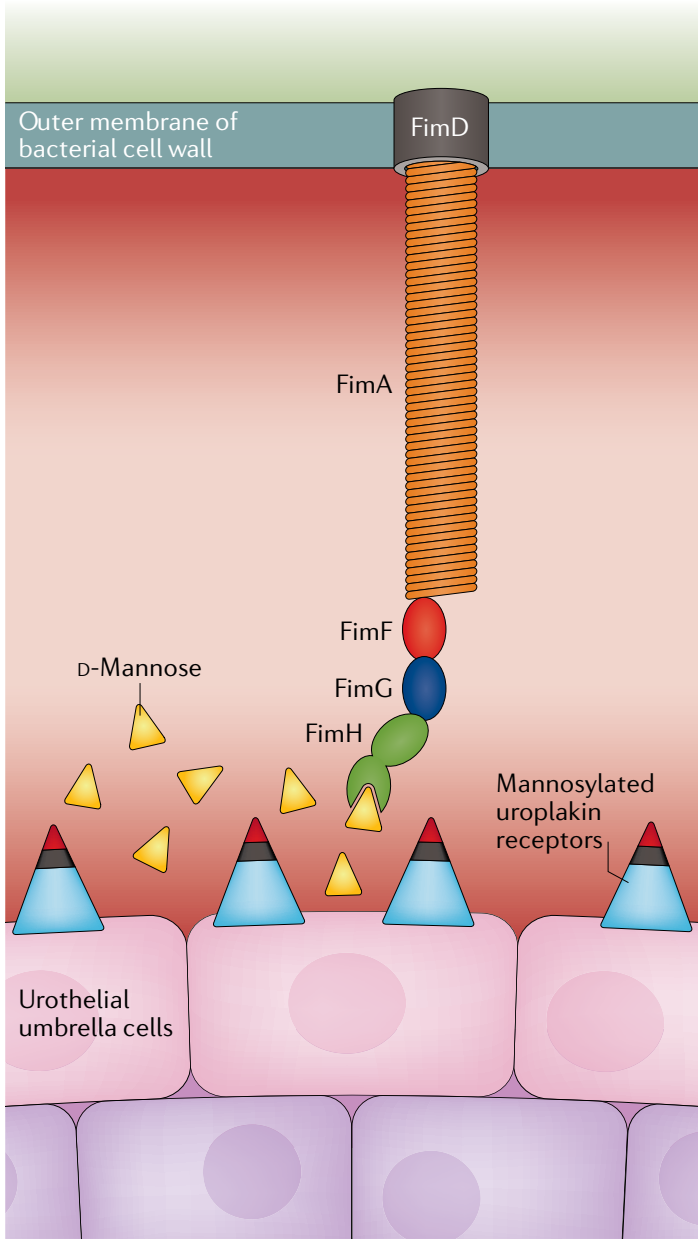

Fig. 7 | D-Mannose prevents binding of bacteria to urothelial cells. D-Mannose is similar in structure to the binding site of urothelial glycoprotein receptors (such as uroplakin) and, therefore, can cause saturation of FimH adhesins and prevent bacteria from binding to urothelial glycoprotein receptors if present in sufficient concentration in the urine.

affinity for FimH than D-mannose. In a mouse model of UPEC colonization created by transurethral inoculation of the human cystitis E. coli isolate UTI89, oral administration of M4284 resulted in significantly reduced intestinal colonization with UPEC in addition to treating UTI compared with mice treated with a vehicle control $(10 \%$ cyclodextrin) $(P<0.05)^{154}$. FimH antagonists have also been shown to increase the susceptibility of UPEC to antibiotics, even in antimicrobial-resistant bacteria ${ }^{153}$. The development of such synthetic mannosides as therapeutic agents has shown promise as novel nonantibiotic treatments for UTI in preclinical studies, and future clinical trials in humans are required before the role of FimH antagonists can be confirmed ${ }^{155}$.

\section{Clinical evidence}

Clinical evidence for D-mannose is lacking, and only one randomized trial of this product exists (BOX 1). In this randomized, prospective, controlled study of 308 women with recurrent UTI (two acute cystitis episodes within 6 months or three within 1 year), participants were randomized to take $\mathrm{D}$-mannose daily ( $2 \mathrm{~g}$ of powder diluted in $200 \mathrm{ml}$ of normal saline (U-tract)), nitrofurantoin $\left(50 \mathrm{mg}\right.$ daily) or no prophylaxis ${ }^{156}$. Median patient age was 49 years, and $47 \%$ of patients were postmenopausal with no significant difference in the number of postmenopausal women between the three groups. Each patient had been treated for an acute UTI with a 1-week course of ciprofloxacin and was confirmed to be free of UTI at the time of inclusion into the study by a MSU culture showing $<10^{3} \mathrm{CFU} / \mathrm{ml}$ of urine. Approximately $77 \%$ of the UTIs were caused by E. coli. During the 6 months following acute UTI, those participants receiving D-mannose or antibiotics had a statistically significant lower risk of recurrent UTI than those who received no treatment $(P<0.0001$ for both treatments compared with no treatment), with an absolute risk reduction of $45 \%$. No statistically significant difference was observed when comparing D-mannose and nitrofurantoin groups. Recurrent acute UTI was seen in $15 \%, 20 \%$ and $61 \%$ in the D-mannose, nitrofurantoin and no prophylaxis arms, respectively. D-Mannose was better tolerated than nitrofurantoin, with $8 \%$ of patients complaining of diarrhoea as the only complaint in the D-mannose group ${ }^{156}$.

D-Mannose compliance has been studied in a small, uncontrolled case series of patients with multiple sclerosis ${ }^{157}$. In this study, compliance was monitored via weekly returns of a usage diary, phone calls from the study team at weeks 1 and 8 of the study and by weighing and counting the D-mannose containers at the study end point (week 16). D-Mannose was well tolerated, and compliance rates with medication were very high (mean 99\%); the most common reason for not taking D-mannose was a failure to remember. This study was not designed to assess efficacy, but reported that D-mannose reduced UTI rate per month by $75 \%$ and $63 \%$ in patients without and with catheters, respectively. However, this group of patients with neurogenic bladders, some of whom were using catheters, was heterogeneous and UTI diagnosis was made on the basis of patient self-testing by urine dipstick alone $e^{157}$. The uncontrolled nature of the study and the small sample size mean that the applicability of its results are limited. However, this investigation highlights the need for well-powered, randomized, placebo-controlled trials of D-mannose.

A combination of nonantibiotic measures might lead to superior results to monotherapy. D-Mannose in conjunction with cranberry extract and lactobacilli in powder form has also been shown to successfully treat acute uncomplicated cystitis and symptoms of UTI in 33 premenopausal women in a small pilot study ${ }^{158}$. In this noncontrolled pilot study, women with acute UTI were given two doses per day of the above combination during the first month and then one sachet per day until the 60th day. Urine dipstick was negative for nitrites and leukocyte esterase in all patients at day 60 . Furthermore, the combination of D-mannose, $\mathrm{N}$-acetylcysteine (a medication that has been shown to reduce bacterial biofilm formation in vitro ${ }^{159}$ ) and Morinda citrifolia (a fruit extract that is thought to have anti-inflammatory and analgesic properties) for 7 days was shown to be equivalent to the daily administration of the antibiotic prulifloxacin for 5 days in preventing UTI after 


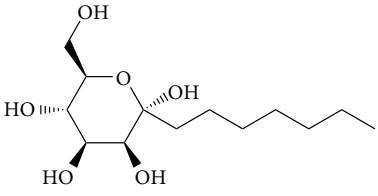

Heptyl $\alpha-D-M a n n o s e$

Fig. 8 | Chemical structure of heptyl- $\alpha-D-m a n n o s e$. Heptyl- $\alpha$-D-mannose is a synthetic mannoside that has an optimized alkyl length for interaction with FimH and, once bound, the bacteria are irreversibly inhibited in their ability to bind to the urothelial surface, resulting in reduced invasion and intracellular bacterial community formation ${ }^{152}$.

urodynamic studies ${ }^{160}$. In this study of 80 patients undergoing urodynamic investigation, 40 were randomized to the combination described above and 40 were randomized to receive antibiotics after the urodynamic study; 10 days after the urodynamic study, patients underwent urine culture. The incidence of UTI was similar in both groups (7.89\% for the antibiotic group and $5.4 \%$ in the combination group; $P=0.671)^{160}$.

These results suggest that $\mathrm{D}$-mannose could be an alternative to antibiotics in treating uncomplicated UTI in women and reduces the risk of recurrence. The evidence shows that D-mannose is well tolerated, but larger randomized studies are required to further validate its use. Assessment of the role of $\mathrm{D}$-mannose in acute treatment versus prophylaxis needs further clarification, as does its role in men with UTI and complex UTIs (such as those associated with neurogenic bladder). The development of synthetic mannosides with much higher affinity for FimH than D-mannose might be a more promising avenue for the prevention of recurrent UTI, as has been suggested in preclinical studies, and clinical trials of these agents are awaited.

\section{Methenamine hippurate}

Methenamine hippurate has been used for decades as a urinary tract antiseptic in treating UTI as it offers the advantages of being bacteriostatic without the development of antimicrobial resistance.

\section{Mechanism of action}

Methenamine, a cyclic hydrocarbon, is hydrolysed in an acid environment into ammonia and formaldehyde according to the reaction:

$$
\mathrm{N}_{4}\left(\mathrm{CH}_{2}\right)_{6}+6 \mathrm{H}_{2} \mathrm{O} \rightarrow 4 \mathrm{NH}_{3}+6 \mathrm{HCHO}
$$

The mechanism of action of methenamine salts in UTIs is thought to be bacteriostatic owing to the production of formaldehyde from hexamine, which occurs in acidic urine ${ }^{161}$. Formaldehyde is thought to be bacteriostatic by inhibiting cell division and by leading to the formation of 1,3-thiazane-4-carboxylic acid, which prevents the synthesis of methionine, an essential metabolite involved in cytoplasmic synthesis. Thus, the action of formaldehyde is to inhibit nuclear and cytoplasmic synthesis, resulting in bacteriostasis ${ }^{162}$. Some studies have recommended using ascorbic acid in addition to methenamine hippurate as a urinary acidifier, as, in vitro, a urinary $\mathrm{pH}$ of $<5.85$ is required to produce the optimal bacteriostatic concentration of formaldehyde from methenamine ${ }^{163}$; whether this acidification is necessary or aids its mode of action is unclear ${ }^{164-166}$.

\section{Clinical evidence}

No clinical trials on methenamine hippurate for UTI have been reported since 2007. The use of methenamine hippurate in treating UTI was the subject of a Cochrane review in 2012 (REF. ${ }^{164}$ ) (BOX 1). In total, 13 studies involving 2,032 participants were included, with the majority of the studies taking place before the year 2000. The dose of methenamine hippurate varied between $1 \mathrm{~g}$ and $4 \mathrm{~g}$ daily, with two studies also using a urinary acidification agent (vitamin $\mathrm{C}$ or sodium acid phosphate) ${ }^{167,168}$. The data pool used reflected a very heterogeneous patient population with cohorts from premenopausal and postmenopausal women, those with recurrent UTI, men undergoing prostate surgery, women after gynaecological surgery, patients with spinal cord injury and those with urinary tract calculi. Approximately half the studies had only a 1-month follow-up duration. Symptomatic UTI was reported in six studies (654 patients), and bacteriuria was reported in eight studies (796 patients). The overall pooled estimates for the major outcome measures (the proportion of patients with symptomatic UTI and the proportion of patients with UTI confirmed by microbiological criteria) were not interpretable because of underlying heterogeneity. However, the subgroup analyses suggested that methenamine hippurate might be beneficial in patients without renal tract abnormalities (symptomatic UTI: RR 0.24, 95\% CI 0.07-0.89; bacteriuria: RR $0.56,95 \%$ CI 0.37-0.83) compared with those with known abnormalities (symptomatic UTI: RR 1.54, 95\% CI 0.38-6.20; bacteriuria: RR 1.29, 95\% CI 0.54-3.07). For short-term treatment duration of $\leq 1$ week, a significant reduction was observed in patients with symptomatic UTI without renal tract abnormalities (RR 0.14, 95\% CI 0.05-0.38). Generally, methenamine hippurate was very well tolerated and the most common adverse event was nausea.

In the UK, a multicentre, noninferiority, randomized trial comparing antibiotic prophylaxis with methenamine hippurate for the management of recurrent UTI (ALTAR trial) has completed recruitment ${ }^{169}$. Primary end points include the incidence of symptomatic antibiotictreated UTI self-reported by participants over the 12-month treatment period and the cost-effectiveness of treatments in the UK National Health Service.

Methenamine hippurate has been shown to be beneficial in the prevention of recurrent UTI, with a low adverse-effect profile and no risk of antimicrobial resistance. Results from the ALTAR trial are awaited, but the use of methenamine hippurate is a very promising nonantibiotic treatment option for patients with recurrent UTI.

\section{Estrogens}

UTIs occur owing to imbalanced interplay between usual host defence and bacterial virulence ${ }^{170}$. Oestrogen hormones are potent regulators of cell growth and 
differentiation ${ }^{171}$. The principal oestrogen secreted by the ovary is $17 \beta$-oestradiol (E2) ${ }^{172}$. Oestrone is also secreted, but the main source of this hormone is extraglandular conversion of androstenedione in peripheral tissues $^{173}$. Hydroxylation of both these hormones leads to formation of oestriol, the main oestrogen that is found in urine $\mathrm{u}^{174}$. Epithelial differentiation and maintenance is predominantly mediated through two major oestrogen receptors (ERs) - ER $\alpha$ and $E R \beta^{175}$. Expression of these receptors is observed in the distal vagina, the trigonal bladder and the epithelial lining and submucosal vascular complex of the urethra ${ }^{174}$. ER $\beta$ predominates in the urinary bladder and ER $\alpha$ in the vagina ${ }^{175}$. Oestrogen sensitivity has also been found in the pubococcygeus and musculature of the pelvic floor ${ }^{176}$. Declining levels of oestrogen after the menopause contribute to chemical and structural changes (such as reduced urinary flow and increased residual volume as well as an increase in vaginal $\mathrm{pH}$ with loss of commensal lactobacilli in the vagina) that increase the risk of UTI ${ }^{175}$. Oestrogen has a key role in modulating the natural defences of the lower urinary tract against UTI. However, the specific mechanisms of this defence are still poorly understood ${ }^{177}$.

\section{Mechanism of action}

In the vagina. Circulating oestrogens in premenopausal women encourage vaginal colonization with lactobacilli ${ }^{178}$. Increased vaginal oestrogen levels have also been shown to increase the proportion of glycogen-producing vaginal epithelial cells, and treatment with depot medroxyprogesterone acetate has been shown to reduce the thickness of the glycogen-bearing epithelial layers, suggesting a role of oestrogens in the promotion of glycogen storage within the vaginal epithelium ${ }^{179}$, allowing lactobacilli to thrive. The conversion by lactobacilli of glycogen into lactic acid, hydrogen peroxide and antimicrobial peptides (such as bacteriocin) maintains a low (acidic) vaginal $\mathrm{pH}(\mathrm{pH}<4.5)$ and inhibits the growth of uropathogens ${ }^{175,180}$. The rise in vaginal $\mathrm{pH}$ (above $\mathrm{pH} 4.5$ ) after the menopause results in reduction in numbers of lactobacilli and an increase in vaginal colonization by gut organisms (such as E. coli). A randomized controlled trial ${ }^{180}$ investigated the effect of intravaginal estriol cream versus placebo on the incidence of UTIs in 93 postmenopausal women with a history of recurrent UTI. A significantly reduced rate of UTI was observed in the estriol group compared with placebo ( 0.5 versus 5.9 episodes per patient-year; $P<0.001)$. Lactobacilli were completely absent in all vaginal cultures at the start of the study but returned in $61 \%$ of patients in the estriol cream group versus none in the placebo group after 1 month of treatment $(P<0.001)$. The mean decrease of vaginal $\mathrm{pH}$ was from 5.5 to 3.8 in the estriol group versus no change with placebo. Vaginal colonization with Enterobacteriaceae also declined from $67 \%$ to $31 \%$ compared with no difference in the placebo group. The authors concluded that the use of intravaginal estriol cream helps to reduce the incidence of UTIs through its effects on the vaginal $\mathrm{pH}$ and microflora. However, 20\% of patients in the estriol group withdrew owing to adverse effects (vaginal irritation, burning or itching), compared with $9 \%$ in the placebo group. Thus, women should be counselled about these possible adverse effects before initiation of therapy in order to improve compliance, as this treatment has been shown to be successful in the prevention of recurrent UTI in postmenopausal women.

In the bladder and the urethra. Oestrogens are known to modulate cell growth and differentiation ${ }^{171}$. The potential effect of oestrogens on antimicrobial peptides and defence mechanisms within the urogenital epithelium has, therefore, been explored. Studies have shown that bacterial contact with urothelial cells results in the release of a cathelicidin antimicrobial peptide LL-37 and its precursor, nCAP-18, into the urine as defence ${ }^{181}$. As LL-37 and nCAP-18 are also expressed by immune cells, levels can be measured in serum. Luthje and colleagues ${ }^{182}$ found that serum cathelicidin levels were significantly reduced in postmenopausal women $(P=0.007)$ and that these positively correlated directly with oestradiol levels (Spearman $r=0.57, P=0.01$ ). To understand whether oestrogen directly affects the expression of antimicrobial peptides, the researchers measured mRNA expression of five different antimicrobial peptides (human $\beta$-defensins 1, 2 and 3; psoriasin; and ribonuclease 7) in exfoliated cells from urine. Comparing samples from postmenopausal women before and after 2 weeks of estradiol supplementation (administered as a vaginal insert of $25 \mu \mathrm{g}$ of estradiol daily), $75 \%$ showed enhanced mRNA expression of at least three peptides and 50\% showed elevated peptide levels in four or all five after estradiol treatment. Unfortunately, cathelicidin expression in urinary cells was low; thus, statistical analysis for this particular antimicrobial was not possible ${ }^{182}$.

The hormonal influence of increased oestrogen levels on the trigone and urethral mucosa also contributes to increased urethral closure pressures and, therefore, improved continence ${ }^{176}$. Atrophy of the vagina, urethra and trigone can also be alleviated with topical estrogen $^{173}$, thereby improving continence and reducing postvoid residual volume. The association between a high postvoid residual volume, incontinence and increased risk of recurrent UTI in the context of low oestrogen levels is well documented ${ }^{41,183}$.

\section{Clinical evidence}

Systemic administration. Oral estrogen therapy in the young postmenopausal woman (age 50-60 years) is often used to avoid menopausal symptoms such as hot flushes, night sweats, vaginal dryness and insomnia. Concurrently, the patient could benefit from the prevention of UTI, osteoporosis and ischaemic heart disease as the risk of these conditions is increased in postmenopausal women ${ }^{184,185}$. In particular, women benefit from improvement in atrophic vaginitis and improved urge incontinence ${ }^{186}$. Absolute contraindications to estrogen therapy include a history of endometrial or breast cancer, thromboembolic disorders (such as deep vein thrombosis or ischaemic stroke) and acute liver disease owing to the risk of worsening cholestasis ${ }^{173}$.

Oral estrogens (with or without progestogens) versus placebo have been studied in the prevention of recurrent UTI in four randomized control studies in a total of 2,798 postmenopausal women ${ }^{187-190}$. The results showed 
that oral estrogens do not reduce the frequency of recurrent UTI (RR 1.08, 95\% CI 0.88-1.33) or increase the number of lactobacilli in the urinary tract of women after treatment ${ }^{191}$. On meta-analysis of two studies that reported changes in vaginal $\mathrm{pH}\left(\mathrm{REFS}^{189,190}\right)$, a considerable decrease in vaginal $\mathrm{pH}$ was observed (from mean pH 6.5 to 5.5 , mean difference -1.00 (95\% CI -1.43 to $-0.57)$ ), with increased adverse effects such as breast tenderness and mild vaginal bleeding in those taking oral estrogens ${ }^{191}$. Thus, preparations for local administration are recommended for the prophylaxis of recurrent UTI instead of systemic administration.

Local administration. Locally administered preparations of estrogen can come as topically applied intravaginal cream, vaginal rings, impregnated pessary rings, vaginal pessaries and vaginal tablets. Estriol at a dose of $0.5 \mathrm{mg}$ is absorbed and excreted equally whether in cream or pessary form ${ }^{192}$, but a Cochrane review concluded that women appeared to favour the vaginal ring for ease of use and overall satisfaction ${ }^{193}$. Two randomized controlled trials have investigated the effects of locally administered estrogen versus placebo in preventing UTI recurrence in women with a history of recurrent UTI (three or more symptomatic UTIs in the previous year) ${ }^{180,194}$. These studies had different methods of estrogen application (comparison of topical estriol cream with placebo cream ${ }^{180}$ or comparison of $2 \mathrm{mg}$ estriol silicone vaginal ring with a no-treatment group $\left.{ }^{194}\right)$. Both trials showed a significant reduction in the proportion of UTIs, with 0.5 versus 5.9 UTI episodes per patient-year with intravaginal estriol cream compared with placebo $(P<0.001)^{180}$, and the cumulative proportion of patients remaining free of UTI was significantly higher with the estriol vaginal ring than placebo $(P=0.008)^{194}$. The results of these trials could not be pooled in a Cochrane meta-analysis owing to heterogeneity between the studies. Adverse effects, such as itching, burning, vaginal discharge and menorrhagia, were more frequent in those using topical estrogen than in the placebo group ${ }^{192}$.

Two randomized controlled trials have compared vaginal estrogens with prophylactic antibiotics. In one study ${ }^{192}$, 171 postmenopausal women with recurrent UTI (three or more symptomatic UTIs in the previous 12 months, or two episodes in the previous 6 months) were treated with an estriol-containing vaginal pessary daily for 2 weeks and then once every 2 weeks for 9 months, with an oral placebo capsule each night. The control group was given a capsule of nitrofurantoin once at night for 9 months with a placebo vaginal pessary daily for 2 weeks. Considerably fewer UTIs were reported in the antibiotic group than in the estriol group (risk ratio 1.30, 95\% CI 1.01-1.68). However, in the other study ${ }^{195}, 45$ postmenopausal women were either part of the estrogen group (intravaginal premarin cream daily for 3 months) or the antibiotic control (oral ofloxacin $600 \mathrm{mg}$ once daily for 3 months). The incidence of UTI was significantly lower in the topical estrogen cream group than in the antibiotic group $(7.4 \%$ compared with $80 \% ; P<0.001)$. Coming to a firm conclusion concerning intravaginal estrogen treatment for prevention of recurrent UTI on the basis of these studies is hampered by the difference in study parameters, the small sample sizes and the unblinded nature of the trials, which increases their risk of bias.

The role of topical estrogen for the prevention of recurrent UTI in postmenopausal women compared with placebo is clearly apparent and is recommended by the European Association of Urology (EAU) guidelines for this population (TABLE 2). However, the outcomes of studies reporting its benefit over antibiotic prophylaxis are mixed. The heterogeneity of studies and small sample sizes means that the overall quality of evidence is low; thus, larger randomized trials comparing topical estrogen with antibiotic prophylaxis are required (BOX 1). The full extent of ER sensitivity, the effect of oestrogen on the urogenital epithelium and the oestrogenic effect on antimicrobial peptides are not well understood and are areas that require further research. Vaginal topical creams seem more effective than rings or pessaries, but consensus on the optimal dosage or prophylaxis duration has yet to be reached.

\section{Intravesical glycosaminoglycans}

The intravesical administration of glycosaminoglycans to treat chronic forms of cystitis, including recurrent bacterial cystitis, has been increasingly studied. The surface of the bladder urothelium is lined by a mucous layer composed of highly negatively charged polysaccharides (glycosaminoglycans and proteoglycans) with a tightly bound water layer on its surface ${ }^{196}$ (FIG. 9). This layer has

Table 2 | European Association of Urology recommendations regarding non-antimicrobial prophylaxis ${ }^{227}$

\begin{tabular}{lll}
\hline Intervention & Grade of recommendation & Notes \\
\hline $\begin{array}{l}\text { Advice on behavioural modifications to reduce } \\
\text { UTI risk }\end{array}$ & C & NA \\
\hline Vaginal oestrogen replacement & A & $\begin{array}{l}\text { Only in postmenopausal women } \\
\text { with recurrent UTI }\end{array}$ \\
\hline $\begin{array}{l}\text { Immunoactive prophylaxis to reduce recurrent } \\
\text { UTI in all age groups }\end{array}$ & A & Based on studies of Uro-Vaxom \\
\hline Probiotics & No recommendation & Further trials are warranted \\
\hline Cranberry & No recommendation & Further trials are warranted \\
\hline D-Mannose & No recommendation & Further trials are warranted \\
\hline Intravesical instillations & No recommendation & Further trials are warranted \\
\hline
\end{tabular}

NA, not applicable; UTI, urinary tract infection. 


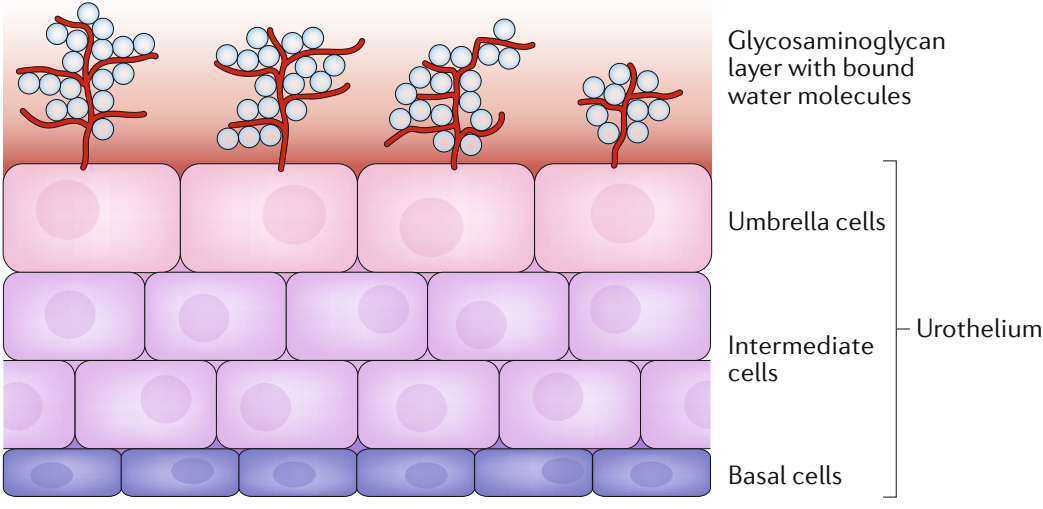

Fig. 9 | Structure of the glycosaminoglygan layer of the bladder wall. The surface of the bladder urothelium is lined by a mucous layer composed of highly negatively charged polysaccharides (glycosaminoglycans and proteoglycans), with a tightly bound water layer on its surface ${ }^{196}$. catalase were measured in four groups of rats: a control group treated only with isotonic saline; untreated rats with bacterial cystitis; rats with bacterial cystitis treated with gentamicin $(4 \mathrm{mg} / \mathrm{kg}$ intramuscularly twice daily for 5 days after $E$. coli inoculation); and infected rats treated with intravesical hyaluronic acid (single dose of $0.5 \mathrm{ml}$ of $0.5 \%$ hyaluronic acid) ${ }^{204}$. Levels of superoxide dismutase and catalase were significantly lower in the untreated group and gentamicintreated group than in the control group $(P<0.01$ and $P<0.05$, respectively), but in the group treated with hyaluronic acid, levels of these antioxidants were no different from the control group, suggesting a role for hyaluronic acid in protecting cells from oxidative stress $^{204}$. Another study showed that the combination of hyaluronic acid and chondroitin sulfate led to greater reductions in bacterial load than with either agent alone and preserved urothelial integrity ${ }^{205}$. This effect is thought to occur because chondroitin sulfate increases the viscosity of hyaluronic acid, thereby improving its mucoadhesive properties and reducing its biodegradation ${ }^{206}$. Subsequent studies have suggested that the glycosaminoglycan layer is not simply a passive layer. This layer has been shown to interact with a wide variety of pathogens (such as herpes simplex virus and Neisseria gonorrhoeae) and has an active role in pathogen attachment to and invasion of host cells $s^{207,208}$. An in vitro study investigated the role of two transmembrane heparan sulfate proteoglycans, syndecan 1 and syndecan 4 , in the uptake of N. gonorrhoeae by epithelial cells (using the cervical cancer cell line HeLa ${ }^{207}$. Overexpression of syndecan 1 and syndecan 4 leads to a threefold and sevenfold increase in $N$. gonorrhoeae invasion, respectively. Deletion of the intracellular domains of syndecan 1 and syndecan 4 reduced the invasion of epithelial cells by $N$. gonorrhoeae, suggesting that the intracellular domains have a crucial role in the process of bacterial invasion, possibly by mediating signal transduction through protein kinase $\mathrm{C}$ and phosphatidylinositol 4,5-bisphosphate. The glycosaminoglycan layer is a promising target for the discovery of new therapeutic agents to prevent UTI, but its role in UTI pathogenesis remains to be fully determined. administration of hyaluronic acid into the bladder led to a significant decrease in positive urine cultures and bladder bacterial growth from $92 \%$ to $20 \%(P<0.05)$ after 1 day, and the effect persisted for 7 days $^{202}$. In terms of a suspected mechanism of action, hyaluronic acid was not found to be directly toxic to $E$. coli when the two were inoculated together, but was observed to coat the urothelial surface, suggesting that it has a barrier role in preventing E. coli and its toxic factors from accessing the underlying urothelial cells ${ }^{202}$. An ex vivo study in mouse bladders using Texas-Red-labelled chondroitin sulfate showed that chondroitin sulfate predominantly binds to areas of damaged urothelium, with minimal binding to undamaged bladder and no penetration into deeper bladder wall layers, again reinforcing its barrier role ${ }^{203}$. A rat study of bacterial cystitis showed that hyaluronic acid also maintained endogenous antioxidant enzymes ${ }^{204}$. In this study, levels of the endogenous antioxidants superoxide dismutase and

\section{Clinical evidence}

Clinical studies evaluating exogenous analogues of the cystitis have been increasing. A number of studies have shown a substantial beneficial effect in preventing recurrent UTI, but the data are predominantly from case series of small numbers of patients; thus, the conclusions of these trials cannot be applied in clinical practice without further confirmation in larger, well-designed, randomized controlled trials ${ }^{209,210}$. One study enrolled 40 women (mean age 35 years) with a history of recurrent UTI (defined as at least three episodes of UTI with a growth of $>10^{3} \mathrm{CFU} / \mathrm{ml}$ of a uropathogen) who received intravesical hyaluronic acid ( $40 \mathrm{mg}$ in $50 \mathrm{ml}$ of phosphate-buffered saline) weekly for 4 weeks and then monthly for 4 months ${ }^{209}$. The results showed that $70 \%$ of these women were free of recurrence at the end of the follow-up period (mean 12.4 months) ${ }^{209}$. glycosaminoglycan layer for the treatment of bacterial 
The mean rate of UTI per patient-year was 4.3 before treatment and 0.3 after treatment $(P<0.001)$. The median time to recurrence after treatment was 498 days compared with 96 days before treatment $(P<0.001)^{209}$. Another study included 20 women (mean age 27.7 years) with recurrent UTI (defined as at least three episodes of uncomplicated cystitis with clinical symptoms and/or a positive culture for each episode, defined as the isolation of $>10^{3} \mathrm{CFU} / \mathrm{ml}$ of urine) who received intravesical hyaluronic acid $(40 \mathrm{mg}$ in $50 \mathrm{ml}$ of phosphate-buffered saline) weekly for 4 weeks and then monthly for 5 months ${ }^{210}$. The results showed a significant reduction in the number of infections per year per patient from 4.99 to $0.56(P<0.001)^{210}$. These results have been supported by two randomized controlled trials investigating a combination of hyaluronic acid and chondroitin sulfate in preventing recurrent UTI in small numbers of patients. In one study, intravesical hyaluronic acid plus chondroitin sulfate was compared with placebo (normal saline), administered weekly for 4 weeks then monthly for 5 months, in 57 women (mean age 34 years) with recurrent UTI (defined as three episodes of symptomatic and microbiologically confirmed UTI in the previous year) ${ }^{211}$. Overall, 28 women were treated with hyaluronic acid and chondroitin sulfate and 29 were randomized to the placebo group. At the 1-year follow-up point, significantly fewer UTIs per patient were observed in the treatment group (mean 0.67) than in the control group (mean 4.19) $(P<0.001)$, and than the mean rate of UTI occurrence in the year before enrolment (4.71). The mean time to UTI recurrence was improved from 52.7 days to 185.2 days, and $48 \%$ of women had not experienced a UTI at 1 year compared with $0 \%$ in the control group. Another randomized trial of 26 women of all ages (mean age 60 years) with recurrent UTI (defined as three episodes of symptomatic and/or microbiologically confirmed UTI in the previous year) compared combined hyaluronic acid and chondroitin sulfate weekly for 4 weeks and then every 2 weeks for 1 month with antibiotic prophylaxis with sulfamethoxazole $200 \mathrm{mg}$ and trimethoprim $40 \mathrm{mg}$ once a week for 6 weeks ${ }^{212}$. A considerable reduction in the mean rate of UTI per patient per year was observed, from 6.3 in the year before treatment to 1.0 at 1 year following treatment in the hyaluronic acid and chondroitin sulfate group, and this was significantly better than antibiotic prophylaxis (mean UTI episodes at 12 months 1.0 in the treatment group versus 2.3 with placebo; $P=0.02$ ). A number of retrospective studies have been performed, using the combination of hyaluronic acid plus chondroitin sulfate for women with a history of recurrent UTI (defined as three episodes of symptomatic and microbiologically confirmed UTI in the previous year). In one study, 157 women of all ages (mean age 54.2) undergoing treatment for a total of 6 months were included. A significant reduction in the mean rate of UTI per patient from 4.13 in the previous year to 0.44 at 1 year after treatment was observed $(P=0.01)^{213}$. A large multicentre study of 174 premenopausal women compared 5 months of treatment with hyaluronic acid plus chondroitin sulfate $(n=98)$ with antibiotic prophylaxis $(n=76)$ with daily sulfamethoxazole $200 \mathrm{mg}$ plus trimethoprim $40 \mathrm{mg}$ for
6 weeks and reported that $36.7 \%$ of patients were free of recurrence in the treatment group compared with $21 \%$ in the antibiotic prophylaxis group $(P=0.03)^{214}$. Another retrospective series divided 69 women into three groups: intravesical hyaluronic acid plus chondroitin sulfate weekly for 4 weeks, every 15 days for 2 months, and then every 30 days for 2 months $(n=22)$; fosfomycin every 10 days for 6 months $(n=23)$; and the combination of hyaluronic acid plus chondroitin sulfate and fosfomycin $(n=24)^{215}$. The results were significant, with only $30.4 \%$ of women free of any recurrence at 6 months in the fosfomycin group compared with $72.7 \%$ in the hyaluronic acid plus chondroitin sulfate group and $75 \%$ in the triple combination group $(P=0.0029)$. The results of the largest study of combination intravesical therapy with hyaluronic acid plus chondroitin sulfate compared with the standard of care to date have been reported ${ }^{216}$. This multi-institutional study included 276 women (mean age 55.2 years in the hyaluronic acid and chondroitin sulfate group and 48.8 years in the standard-of-care group). The hyaluronic acid plus chondroitin sulfate group had a $49 \%$ reduction (OR $0.51,95 \%$ CI $0.27-0.96$ ) in the risk of an MSU-confirmed UTI compared with the standardof-care group. However, once a patient had a recurrence in the hyaluronic acid plus chondroitin sulfate group, no evidence of improvement in terms of total number of recurrences or time to first recurrence was observed compared with the standard-of-care group. The beneficial effects of intravesical hyaluronic acid plus chondroitin sulfate therapy were suggested to improve with more than five instillations. However, this study has limitations, including a non-standardized treatment regimen for the intravesical therapy, no specification for the standardof-care treatment and its retrospective nature. As a result, the conclusions of this trial must be interpreted with caution, and further studies are warranted to determine the clinical benefit of intravesical hyaluronic acid and chondroitin sulfate in the prevention of recurrent UTI using standardized treatment regimens (BOX 1).

These results seem promising, but some limitations must be acknowledged. The majority of data are from small, retrospective series, the intravesical formulation used varies between studies and the optimal frequency and duration of therapy have not been standardized. Furthermore, no data for men with recurrent UTI have been reported and, therefore, study in this population is warranted. Another concern relates to the cost of the treatment. The estimated cost of a course of intravesical hyaluronic acid plus chondroitin sulfate over a 12-month period is substantially higher than for a 6-month course of antibiotic prophylaxis $(€ 1,500$ versus $€ 30)^{217}$. However, this cost must be balanced against the benefits in terms of preventing antimicrobial resistance and antibiotic-related adverse effects. Studies of patient satisfaction and patient-reported outcomes are also warranted in order to assess the place of this option in the treatment armamentarium. Further research into the mechanisms by which uropathogenic bacteria interact with the glycosaminoglycan layer will lead to the development of novel and more efficacious treatments for the prevention of recurrent UTI. 


\section{Immunostimulants and vaccines}

The effective development of vaccines against UTIs is limited by factors such as a lack of natural protective immunity in humans after UTI, a lack of induction of antibodies in the bladder, the diversity of pathogens and the desire to avoid inducing a pathological immune response to colonizing bacteria in the gut. Infection in the kidney might lead to the development of antibodies, but this adaptive response seems to be lacking in bladder infections ${ }^{218}$. Despite these limitations, multiple promising vaccines and immunostimulants have been trialled in mice, rats and non-human primates, and a small number have progressed to trials in humans ${ }^{219}$ (TABLE 3).

\section{Immunostimulants studied in humans}

Immunostimulants are believed to exert their effects through activation of both the innate and adaptive immune systems. Following ingestion, immunostimulants have been shown to activate dendritic cells, neutrophils and $\mathrm{T}$ helper cells. This activation results in activation of $\mathrm{T}$ lymphocytes and $\mathrm{B}$ lymphocytes, and the subsequent release of IgA results in immunoprotection $^{220,221}$. One of the most widely studied immunostimulants currently available is an oral capsule, OM-89 (Uro-Vaxom), which is made from an extract of 18 strains of heat-killed UPEC. A newer preparation, OM-89-S, containing lysates of the same 18 strains of heat-killed UPEC (also known as Uro-Vaxom) is manufactured by a different lysis process (modified alkaline lysis rather than autolysis), which destroys the lipopolysaccharide cell wall of Gram-negative bacteria and modifies bacterial antigens, thereby maintaining their antigenic potential. Stimulation of the host immune system occurs via the mucosa-associated lymphoid tissue lining the gastrointestinal, respiratory and genitourinary tracts, leading to an increase in the concentration of bacteria-specific antibodies ${ }^{222}$. In immunodeficient mouse models, administration of Uro-Vaxom (OM-89) was shown to cause increased levels of IgG and IgA specific to the $E$. coli strains in the extract, with significant changes in bladder IFN $\gamma$ and IL-6 levels ${ }^{223,224}$. A systematic review of four randomized, placebocontrolled trials investigating OM-89 that included 891 patients with recurrent UTI (three episodes in the previous year) showed that this immunomodulatory preparation had significant efficacy as a prophylactic agent (RR 0.61, 95\% CI 0.48-0.78) ${ }^{96}$ (BOX 1). However, a subsequent large trial of 451 patients with recurrent UTI (mean age 43.90 years) did not demonstrate benefit of the use of OM-89-S as prophylaxis, and, although it also failed to demonstrate efficacy of nitrofurantoin, this result could be because of increasing rates of antibiotic resistance rather than a failure of trial methodology ${ }^{225}$. Uro-Vaxom (OM-89) is recommended in EAU guidelines (TABLE 2) for immunoprophylaxis in women with recurrent uncomplicated UTI on the basis of a metaanalysis of randomized trials ${ }^{226}$, although these guidelines do not refer to the OM-89-S trial, which might alter this recommendation ${ }^{227}$. It seems that the use of OM-89 Uro-Vaxom might reduce prescribing of antibiotics, which alone could justify its use, as it seems to be well tolerated $^{96,225}$. Both OM-89 and OM-89-S include only strains of UPEC, in contrast to other immunostimulants, which tend to include a wider range of uropathogens; however, if efficacious against UPEC alone, it would still have a clear role in UTI management. Further research is required given the results of the OM-89-S trial ${ }^{225}$.

Strovac (also known as Solco-Urovac) comprises ten strains of heat-killed uropathogens (six serotypes of UPEC, P. vulgaris, K. pneumoniae, Morganella morganii and $E$. faecalis) administered by intramuscular injection. This product was subsequently replaced by a vaginal preparation known as Urovac vaginal vaccine owing to considerable adverse reactions at the administration site ${ }^{228}$. A meta-analysis of three randomized, placebo-controlled phase II studies consisting of 220 women (age range 18-74 years) demonstrated a modest effect that supports the need for further investigation of this immunostimulant (RR 0.81, 95\% CI 0.68-0.96) ${ }^{96}$ (BOX 1). Benefits were highest in those patients receiving booster doses of the vaccine at monthly intervals ${ }^{229,230}$, but data are from small studies. Large, randomized phase III trials are required to establish the efficacy of this therapeutic option.

A sublingual preparation, Uromune (MV140), comprises selective strains of inactivated uropathogens (E. coli, K. pneumoniae, P. vulgaris and E. faecalis) (BOX 1). The mechanism of action is not clearly understood, but this preparation has been shown to prime dendritic cells to generate $T$ helper $1\left(T_{H} 1\right)$ cells, $T_{H} 17$ cells and IL-10-secreting $\mathrm{T}$ cells ${ }^{231}$. This vaccine has demonstrated efficacy in cohort studies, with a reported absolute risk reduction of UTI of $90 \%{ }^{232}$, and was well tolerated in a small cohort study in the $\mathrm{UK}^{233}$. In the earliest study of Uromune in women with recurrent UTI, 159 women were treated with Uromune via two puffs of $100 \mu \mathrm{l}$ each ( $10^{8}$ bacteria per puff) daily for 3 months (mean age 47.7 years, range $16-85$ years) and 160 women received daily sulfamethoxazole $200 \mathrm{mg}$ and trimethoprim $40 \mathrm{mg}$ for a period of 6 months ${ }^{234}$. A significant reduction in the mean number of UTIs at 3 months was seen with

Table 3 | Characteristics of most commonly studied vaccines in humans for urinary tract infection prophylaxis

\begin{tabular}{|c|c|c|}
\hline Vaccine & Route of administration & Strains of uropathogen \\
\hline $\begin{array}{l}\text { OM-89 and OM-89-S } \\
\text { (Uro-Vaxom) }\end{array}$ & Oral capsule & Eighteen serotypes of UPEC \\
\hline Solco-Urovac & $\begin{array}{l}\text { Vaginal pessary, cream or } \\
\text { suppository }\end{array}$ & $\begin{array}{l}\text { Six serotypes of UPEC, Proteus mirabilis, Morganella } \\
\text { morganii, Klebsiella pneumoniae and Enterococcus } \\
\text { faecalis }\end{array}$ \\
\hline Uromune (MV140) & Sublingual spray & $\begin{array}{l}\text { Escherichia coli, K. pneumoniae, Proteus vulgaris and } \\
\text { E. faecalis }\end{array}$ \\
\hline
\end{tabular}


Uromune compared with antibiotics ( 0.36 versus 1.60 ; $P<0.0001)$, and this reduction was also seen at the 15 -month follow-up point $(P<0.0001)$. A larger retrospective series of women with recurrent UTI compared 360 women (mean age 60 ) treated with a 3 -month course of Uromune with 339 women (mean age 59) treated with a 6-month course of daily antibiotic prophylaxis (sulfamethoxazole and trimethoprim or nitrofurantoin $)^{232}$. At 12 months, only $9.7 \%$ of women treated with Uromune had developed a UTI compared with $100 \%$ of those treated with antibiotics $(P<0.0001)$. These promising results have been supported by a UK cohort study of 77 women with recurrent UTI (mean age 56 years) treated with a 3-month course of Uromune ${ }^{233}$. All women had a minimum of three UTIs in the 12-month period before enrolment. At the 12-month follow-up point, $78 \%$ had no further $\mathrm{UTI}^{233}$. The results of a currently recruiting placebo-controlled trial (NCT02543827) are awaited with interest ${ }^{235}$. Urostim (E. coli, K. pneumoniae, P. vulgaris and E. faecalis) and Urvakol (E. coli, P. aeruginosa, $P$. vulgaris and E. faecalis) are whole-bacterial-cell vaccines that are in development and are administered as tablets containing mixtures of inactivated uropathogens. One study investigated the immune response to $50 \mathrm{mg}$ Urostim daily for 3 months in 35 patients ( 33 female and 2 male) with chronic pyelonephritis ${ }^{236}$. Blood and saliva samples were collected at 30 and 90 days after administration of Urostim, and an improvement was observed in both nonspecific and specific lymphoproliferative responses following Urostim treatment ${ }^{236}$. However, neither vaccine has yet become established for clinical use $\mathrm{e}^{219}$.

\section{Novel vaccines under investigation}

In addition to preparations based on mixtures of uropathogens trialled in humans, candidate vaccine antigens with multiple sites of action of UPEC virulence factors have been tested in mouse models of UTI (BOX 1). As UPEC invading the bladder harness chaperoneusher pathway pili to adhere to the bladder wall, various approaches have targeted these chaperone-usher pathways via immunization. These pathways provide attractive vaccine targets, as the induction of antibodies against adhesins to prevent host-bacteria interactions and prevent bacterial colonization of the bladder is plausible. Type 1 pili have a critical role in the development of UTI, and the type 1 pilus tip adhesin FimH has been harnessed as a putative vaccine ${ }^{237}$. A study in eight cynomolgus monkeys investigated the effect of intramuscular administration of $100 \mu \mathrm{g}$ of FimCH adhesin-chaperone complex mixed with MF59 adjuvant compared with MF59 adjuvant alone ${ }^{237}$. All monkeys were infected with an inoculum containing $10^{8}$ type-1-piliated E. coli 18 days after final immunization, and urine samples were obtained at days 2-14. Results showed that three of four monkeys treated with the vaccine had no bacteria or pyuria, compared with all four of the control monkeys, which had pyuria and a positive urinary growth of E. coli ${ }^{237,238}$. An alternative approach to improve antibody responses is fusion of FimH to the flagellin FliC (a TLR5 agonist of UPEC) using lipopolysaccharide-derived monophosphoryl lipid A (MPA) as an adjuvant ${ }^{239}$.
In this study, 16 mice were immunized with the fused FimH-FliC protein - 16 with FimH alone and 16 with FimH admixed with FliC. The fused FimH-FliC protein induced significantly higher humoral (IgG1 and IgG2a; $P=0.002)$ and cellular (IFN $\gamma$ and IL-4; $P<0.01$ ) immune responses than with FimH alone or FimH admixed with FliC. However, results of studies in humans are required.

$\mathrm{P}$ pili are an alternative to type 1 pili in vaccine approaches, with promising results of immunization with $\mathrm{P}$ fimbriae in the prevention of pyelonephritis demonstrated in cynomolgus monkeys ${ }^{240}$. In this study, monkeys were intraperitoneally vaccinated with $100 \mu \mathrm{g}$ purified PapDG protein before inoculation with $1 \times 10^{8}$ CFU of pyelonephritogenic E. coli. Vaccinated monkeys did not have any histological evidence of pyelonephritis, with significantly lower levels of neutrophilic infiltrate, scarring and interstitial fibrosis than unvaccinated monkeys $(P=0.007)^{240}$. However, studies in humans are lacking. A number of other approaches are promising in animal studies and might, in due course, progress to trials in humans. In a mouse model of chronic UTI, 40 mice were immunized with $10 \mu \mathrm{g}$ of another UPECassociated pilus, Dr fimbrial antigen, and given a booster injection 4 weeks later ${ }^{241}$, and 40 control mice were given placebo (phosphate-buffered saline) ${ }^{241}$. Mice were then inoculated with $E$. coli by intravesical instillation, and in the first week after infection, eight mice (20\%) in the group of nonvaccinated animals died, in contrast to one mouse (2\%) in the group immunized with Dr fimbrial antigen $(P<0.05)$. Another study has investigated immunization against the EbpA pilus, a pilus that contributes to catheter-associated biofilm formation by E. faeca$l i s^{242}$. In this study, mice were immunized with purified EbpA and had booster immunizations at weeks 4 and 8 $\left(\right.$ REF. $\left.^{243}\right)$. Mice were then implanted with catheters and inoculated with $2 \times 10^{7} \mathrm{CFU}$ of $E$. faecalis, and vaccination significantly reduced bacterial colonization in the bladder and on the catheter compared with control mice $(P<0.0005)^{242}$. Another promising vaccine target are the siderophore systems, as UPEC require iron for bacterial growth and colonization ${ }^{243}$. In a mouse model of ascending UTI, intranasal immunization with the UPEC iron receptor, yersiniabactin receptor (FyuA), was found to significantly reduce bacterial colonization $(P=0.0018)^{219,243}$. Although promising, human studies of these newer vaccines are required to determine their utility in clinical practice.

Other virulence factors that have been targeted for vaccine development include toxins produced by uropathogenic organisms. A conjugate vaccine incorporating exoprotein A from $P$. aeruginosa and four O-antigens from selected extraintestinal pathogenic E. coli (ExPEC) serotypes (O1, O2, O6 and O25b) was administered to rabbits ( $2 \mu \mathrm{g}$ or $20 \mu \mathrm{g}$ per O-antigen, subcutaneously), mice $(0.2 \mu$ g or $2 \mu \mathrm{g}$ per $\mathrm{O}$-antigen, subcutaneously) and rats $(0.4 \mu \mathrm{g} \text { or } 4 \mu \mathrm{g} \text { per O-antigen, intramuscularly })^{244}$. Significant antigen-specific IgG responses were observed in all animal models $(P<0.05)^{244}$. In the first-in-human phase Ib randomized clinical trial of a single intramuscular injection of ExPEC4V or placebo in healthy adult women (93 women treated with the vaccine and 95 treated with placebo), the vaccine was tolerated well and 
led to the induction of functional antibodies ${ }^{245}$. Despite these antibody responses, no efficacy was demonstrated against vaccine-specific serotype UTIs ( 0.149 mean episodes versus 0.146 mean episodes; $P=0.522$ ), but significantly fewer UTIs caused by $E$. coli of any serotype were seen in the vaccine group than in the placebo group ( 0.207 mean episodes versus 0.463 mean episodes; $P=0.002)^{245}$; thus, this approach shows promise (for example, UTIs overall were reduced in this study), but its efficacy as a vaccine has not been established.

In conclusion, many approaches to immunostimulation and vaccination to prevent UTIs show promise, but a clearly effective vaccine is still lacking. As our antibiotic prophylactic options reduce, hopefully an immunostimulant, vaccine or live attenuated $E$. coli strain will soon become established as effective in preventing the considerable morbidity associated with the recurrence of UTI.

\section{Competitive inoculation}

Colonization with less-pathogenic strains of UPEC (such as E. coli 83972, an E. coli strain that fails to express virulence factors associated with symptomatic UTI) is also under investigation to prevent recurrent UTI (BOX 1). This approach seems of particular utility in those who are susceptible owing to external factors, such as longterm catheter use and those in whom colonization can be effectively established owing to incomplete bladder emptying $^{246,247}$. E. coli 83972 was isolated from a child with asymptomatic bacteriuria and has been trialled in those with spinal cord injury with a history of recurrent $\mathrm{UTI}^{246}$. In this study, 65 patients with neurogenic bladder after spinal cord injury and a history of recurrent UTI were randomized in a 3:1 ratio to receive either intravesical instillation of $E$. coli or sterile saline ${ }^{246}$. A total of 27 patients were included in the final analysis (17 in the experimental group and 10 in the placebo group), and the mean number of UTIs was reduced in the experimental group (0.50 UTIs per patient-year compared with 1.68 with placebo; $P=0.02)^{246}$. A double-blind randomized crossover trial in patients with incomplete bladder emptying and recurrent UTI investigated the intravesical instillation of E. coli 83972 compared with placebo (saline) ${ }^{247}$. Patients treated with E. coli had a longer time to first UTI than the placebo group (median 11.3 versus 5.7 months; $P=0.0129$ ), and following instillation of E. coli, patients had significantly fewer UTIs than before the $E$. coli instillation ( 13 versus 35 episodes of UTI; $P=0.009)^{247}$. However, colonization is not established in those who have complete bladder emptying; thus, future work should aim to mutate a strain that contains fimbriae enabling adherence. In addition, in some patients treated with E. coli 83972, symptomatic UTI occurred that might have been related to the E. coli 83972 in the absence of functional virulence factors ${ }^{248}$. This observation suggests that asymptomatic carriage of bacteria might lead to an as-yet undefined host immune response that results in the development of symptoms of UTI.

The use of less-pathogenic strains of uropathogenic bacteria to compete with and prevent colonization and infection with disease-causing organisms seems promising, but larger randomized trials in women with recurrent UTI are required to assess the efficacy, safety and cost-effectiveness of this approach.

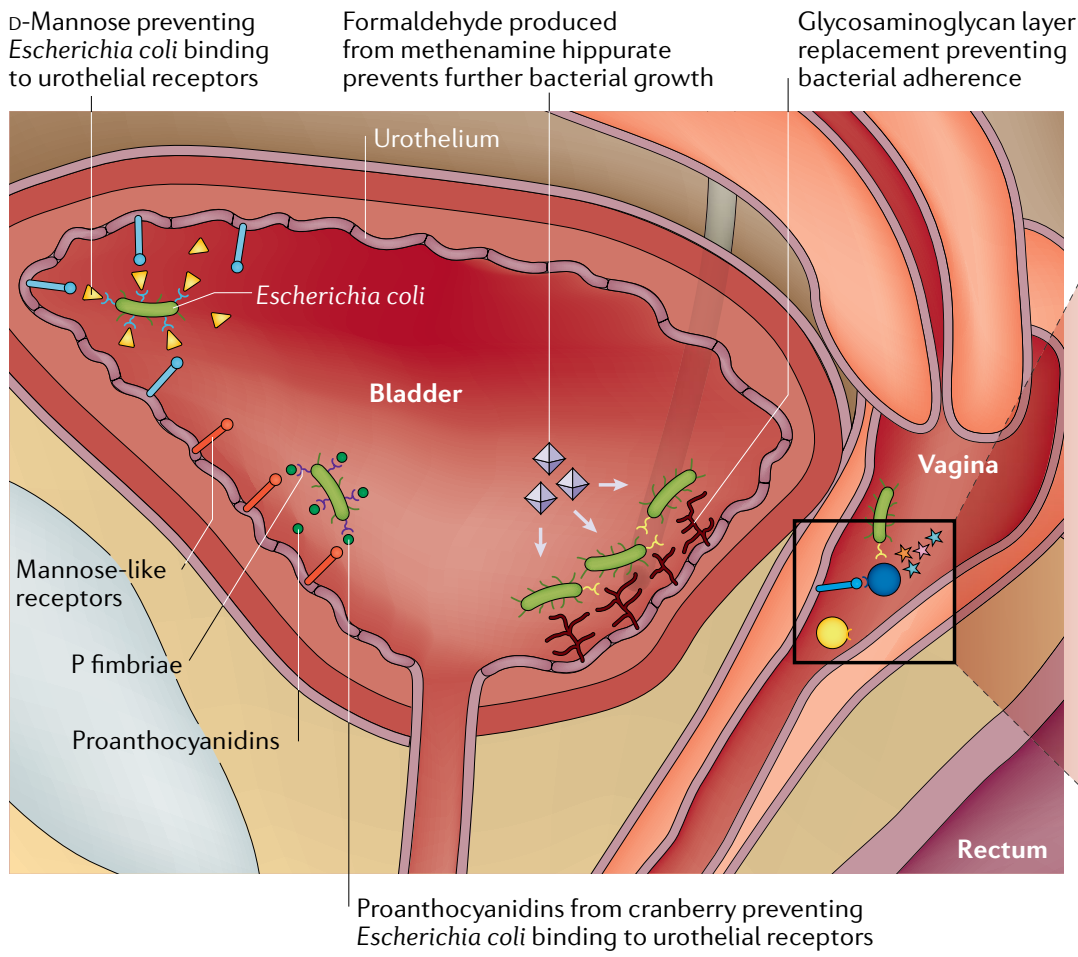

Lactobacilli preventing binding of Escherichia coli to vaginal epithelial adhesion receptors

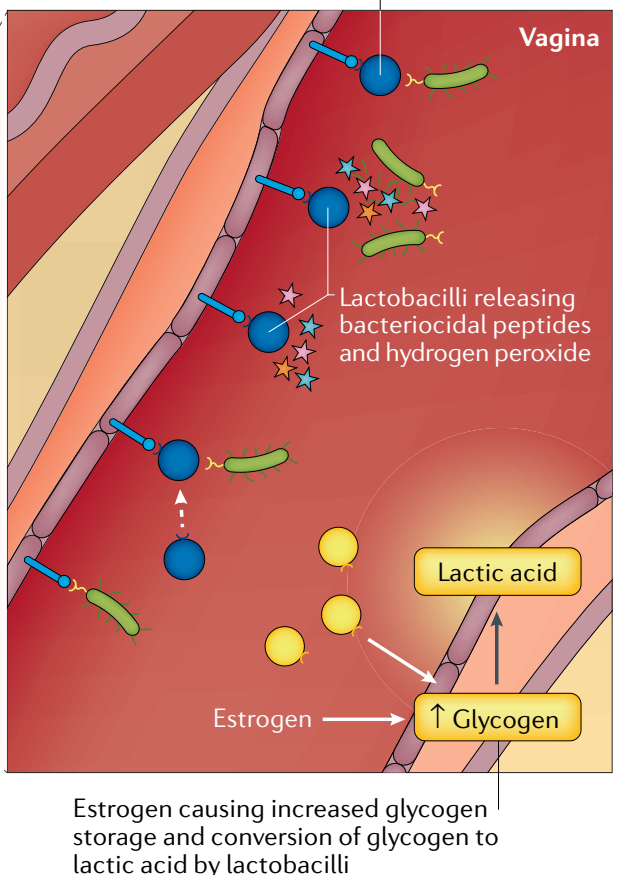

Fig. 10 | Nonantibiotic interventions for urinary tract infection. Nonantibiotic interventions for urinary tract infection (UTI) seem promising, and a combination of these agents might be the most effective way to reduce the rate of recurrent UTI without resorting to antimicrobial use. 


\section{Conclusions}

The nonantibiotic interventions for UTI discussed in this Review seem promising (FIG. 10), but research needs to be improved into the nonantibiotic treatment and prophylaxis of recurrent UTI. Large double-blind, placebo-controlled, randomized studies are required to provide high-level evidence of efficacy for the agents discussed as heterogeneity of the current data is the limiting factor for all agents. A combination of these agents might be the most effective way to reduce the rate of recurrent UTI without resorting to antimicrobial use, and, again, this approach warrants further investigation. Studies of combination therapies in specific patient populations (such as premenopausal women, postmenopausal women, and men) are also needed to target these treatments in the optimal way. Research into the underlying molecular mechanisms of bacterial adherence and invasion seem most promising and could lead to the identification of novel targets for drug development, and many potential therapies are in preclinical development. The aim of these strategies is to reduce the crisis of antimicrobial resistance and pave the way for a new era in the management of recurrent UTI.

Published online 25 October 2018
1. Stamm, W. E. \& Norrby, S. R. Urinary tract infections: disease panorama and challenges. J. Infect. Dis. 183, S1-S4 (2001).

2. Laupland, K. B., Ross, T., Pitout, J. D., Church, D. L. \& Gregson, D. B. Community-onset urinary tract infections: a population-based assessment. Infection 35, 150-153 (2007)

3. Mabeck, C. E. Treatment of uncomplicated urinary tract infection in non-pregnant women. Postgrad. Med. J. 48, 69-75 (1972)

4. Foxman, B. Epidemiology of urinary tract infections: incidence, morbidity, and economic costs. Am. J. Med 113 (Suppl 1A), 5s-13s (2002).

5. Nicolle, L. E. Urinary tract infection. Crit. Care Clin. 29, 699-715 (2013).

6. Loveday, H. P. et al. epic3: national evidence-based guidelines for preventing healthcare-associated infections in NHS hospitals in England. J. Hosp. Infect 86, S1-S70 (2014).

7. Flores-Mireles, A. L., Walker, J. N., Caparon, M. \& Hultgren, S. J. Urinary tract infections: epidemiology, mechanisms of infection and treatment options. Nat. Rev. Microbiol. 13, 269-284 (2015).

8. Ellis, A. K. \& Verma, S. Quality of life in women with urinary tract infections: is benign disease a misnomer? J. Am. Board Fam. Pract. 13, 392-397 (2000).

9. Foxman, B. \& Frerichs, R. R. Epidemiology of urinary tract infection: I. Diaphragm use and sexual intercourse. Am. J. Publ. Health 75, 1308-1313 (1985).

10. Akkerman, A. E., Kuyvenhoven, M. M., Verheij, T. J. $\&$ van Dijk, L. Antibiotics in Dutch general practice: nationwide electronic GP database and national reimbursement rates. Pharmacoepidemiol. Drug Saf. 17, 378-383 (2008).

11. European Centre for Disease Prevention and Control. ECDC/EMEA Joint Technical Report. The bacterial challenge: time to react (ECDC, 2009)

12. Llor, C. \& Bjerrum, L. Antimicrobial resistance: risk associated with antibiotic overuse and initiatives to reduce the problem. Ther. Adv. Drug Safety 5 , 229-241 (2014).

13. Gupta, K. et al. Inverse association of $\mathrm{H} 2 \mathrm{O} 2$-producing lactobacilli and vaginal Escherichia coli colonization in women with recurrent urinary tract infections. J. Infect. Dis. 178, 446-450 (1998).

14. Foxman, B. et al. Uropathogenic Escherichia coli are more likely than commensal $E$. coli to be shared between heterosexual sex partners. Am. J. Epidemiol. 156, 1133-1140 (2002).

15. Mulvey, M. A., Schilling, J. D. \& Hultgren, S. J. Establishment of a persistent Escherichia coli reservoir during the acute phase of a bladder infection. Infect. Immun. 69, 4572-4579 (2001).

16. Schilling, J. D., Lorenz, R. G. \& Hultgren, S. J. Effect of trimethoprim-sulfamethoxazole on recurrent bacteriuria and bacterial persistence in mice infected with uropathogenic. Escherichia coli. Infect. Immun. 70, 7042-7049 (2002)

17. Rosen, D. A., Hooton, T. M., Stamm, W. E., Humphrey, P. A. \& Hultgren, S. J. Detection of intracellular bacterial communities in human urinary tract infection. PLoS Med. 4, e329 (2007)

18. World Health Organisation. Antimicrobial resistance: global report on surveillance 2014 (WHO, 2014)

19. Bryce, A. et al. Global prevalence of antibiotic resistance in paediatric urinary tract infections caused by Escherichia coli and association with routine use of antibiotics in primary care: systematic review and meta-analysis. BMJ 352, i939 (2016).
20. Johnson, J. R., Johnston, B., Clabots, C. Kuskowski, M. A. \& Castanheira, M. Escherichia coli sequence type ST131 as the major cause of serious multidrug-resistant $E$. coli infections in the United States. Clin. Infect. Dis. 51, 286-294 (2010)

21. Wang, Y. et al. Prevalence, risk factors, outcomes, and molecular epidemiology of mcr-1-positive Enterobacteriaceae in patients and healthy adults from China: an epidemiological and clinical study. Lancet Infect. Dis. 17, 390-399 (2017).

22. Blair, J. M., Webber, M. A., Baylay, A. J., Ogbolu, D. O $\&$ Piddock, L. J. Molecular mechanisms of antibiotic resistance. Nat. Rev. Microbiol. 13, 42-51 (2015).

23. Van Boeckel, T. P. et al. Global antibiotic consumption 2000 to 2010: an analysis of national pharmaceutica sales data. Lancet Infect. Dis. 14, 742-750 (2014).

24. Munita, J. M. \& Arias, C. A. Mechanisms of antibiotic resistance. Microbiol. Spectr. https://doi.org/10.1128/ microbiolspec.VMBF-0016-2015 (2016)

25. Abraham, E.P. \& Chain, E. An enzyme from bacteria able to destroy penicillin. 1940. Rev. Infect. Dis. 10, 677-678 (1988).

26. Logan, L. K. \& Weinstein, R. A. The epidemiology of carbapenem-resistant Enterobacteriaceae: the impact and evolution of a global menace. J. Infect. Dis. 215, S28-S36 (2017).

27. Tangden, T., Adler, M., Cars, O., Sandegren, L. $\&$ Lowdin, E. Frequent emergence of porin-deficient subpopulations with reduced carbapenem susceptibility in ESBL-producing Escherichia coli during exposure to ertapenem in an in vitro pharmacokinetic model. J. Antimicrob. Chemother. 68, 1319-1326 (2013)

28. Adler, M., Anjum, M., Andersson, D. I. \& Sandegren, L. Influence of acquired $\beta$-lactamases on the evolution of spontaneous carbapenem resistance in. Escherichia coli. J. Antimicrob. Chemother. 68 51-59 (2013)

29. Ma, L. et al. Widespread dissemination of aminoglycoside resistance genes $a r m A$ and $r m t B$ in Klebsiella pneumoniae isolates in Taiwan producing CTX-M-type extended-spectrum $\beta$-lactamases. Antimicrob. Agents Chemother. 53, 104-111 (2009).

30. Sanchez, G. V., Baird, A. M., Karlowsky, J. A Master, R. N. \& Bordon, J. M. Nitrofurantoin retains antimicrobial activity against multidrug-resistant urinary Escherichia coli from US outpatients. J. Antimicrob. Chemother. 69, 3259-3262 (2014).

31. Russell, G. Antibiotic resistance in children with E coli urinary tract infection. BMJ 352, i1399 (2016).

32. Heinemann, C. \& Reid, G. Vaginal microbial diversity among postmenopausal women with and without hormone replacement therapy. Can. J. Microbiol. 51 , 777-781 (2005)

33. Falagas, M. E., Rafailidis, P. I. \& Makris, G. C. Bacterial interference for the prevention and treatment of infections. Int. J. Antimicrob. Agents 31, 518-522 (2008).

34. Chan, R. C., Reid, G., Irvin, R. T., Bruce, A. W. \& Costerton, J. W. Competitive exclusion of uropathogens from human uroepithelial cells by Lactobacillus whole cells and cell wall fragments. Infect. Immun. 47, 84-89 (1985).

35. Hooton, T. M. et al. A prospective study of risk factors for symptomatic urinary tract infection in young women. N. Engl. J. Med. 335, 468-474 (1996).

36. Hooton, T. M., Roberts, P. L. \& Stamm, W. E. Effects of recent sexual activity and use of a diaphragm on the vaginal microflora Clin. Infect. Dis. 19, 274-278 (1994).

37. Xie, J., Foxman, B., Zhang, L. \& Marrs, C. F. Molecula epidemiologic identification of Escherichia coli genes that are potentially involved in movement of the organism from the intestinal tract to the vagina and bladder. J. Clin. Microbiol. 44, 2434-2441 (2006).

38. Scholes, D. et al. Risk factors for recurrent urinary tract infection in young women. J. Infect. Dis. 182 1177-1182 (2000).

39. Moore, E. E. et al. Sexual intercourse and risk of symptomatic urinary tract infection in postmenopausal women. J. Gen. Intern. Med. 23 595-599 (2008).

40. Foxman, B. et al. Urinary tract infection among women aged 40 to 65 : behavioral and sexual risk factors. J. Clin. Epidemiol. 54, 710-718 (2001).

41. Raz, R. et al. Recurrent urinary tract infections in postmenopausal women. Clin. Infect. Dis. 30, 152-156 (2000).

42. Lema, V. M. Urinary tract infection in young healthy women following heterosexual anal intercourse: case reports. Afr. J. Reproductive Health 19, 134-139 (2015).

43. Coull, N., Mastoroudes, H., Popert, R. \& O’Brien, T. S. Redefining urological history taking - anal intercourse as the cause of unexplained symptoms in heterosexuals. Ann. R. Coll. Surg. Engl. 90, 403-405 (2008).

44. Gupta, K., Hillier, S. L., Hooton, T. M., Roberts, P. L. \& Stamm, W. E. Effects of contraceptive method on the vaginal microbial flora: a prospective evaluation. J. Infect. Dis. 181, 595-601 (2000).

45. Handley, M. A., Reingold, A. L., Shiboski, S. \& Padian, N. S. Incidence of acute urinary tract infection in young women and use of male condoms with and without nonoxynol-9 spermicides. Epidemiology 13, 431-436 (2002).

46. Su, S. B., Wang, J. N., Lu, C. W. \& Guo, H. R. Reducing urinary tract infections among female clean room workers. J. Womens Health 15, 870-876 (2006).

47. Foxman, B. \& Chi, J. W. Health behavior and urinary tract infection in college-aged women. J. Clin. Epidemiol. 43, 329-337 (1990).

48. Adatto, K., Doebele, K. G., Galland, L. \& Granowetter, L. Behavioral factors and urinary tract infection. JAMA 241, 2525-2526 (1979).

49. Nygaard, I. \& Linder, M. Thirst at work - an occupational hazard? Int. Urogynecol. J. Pelvic Floor Dysfunct. 8, 340-343 (1997).

50. Eckford, S. D., Keane, D. P., Lamond, E., Jackson, S. R. $\&$ Abrams, P. Hydration monitoring in the prevention of recurrent idiopathic urinary tract infections in pre-menopausal women. Br. J. Urol. 76, 90-93 (1995).

51. Murphy, F. J., Zelman, S. \& Mau, W. Ascorbic acid as a urinary acidifying agent. 2 . Its adjunctive role in chronic urinary infection. J. Urol. 94, 300-303 (1965).

52. McDonald D. F \& Murphy, G. P. Bacteriostatic and acidifying effects of methionine, hydrolyzed casein, and AA on the urine. N. Engl. J. Med. 261, 803-805 (1959).

53. Castello, T., Girona, L., Gomez, M. R., Mena Mur, A \& Garcia, L. The possible value of ascorbic acid as a prophylactic agent for urinary tract infection. Spinal Cord 34, 592-593 (1996).

54. Peng, M. M., Fang, Y., Hu, W. \& Huang, Q. The pharmacological activities of compound salvia 
plebeia granules on treating urinary tract infection. J. Ethnopharmacol. 129, 59-63 (2010).

55. Yan, D., Jin, C., Xiao, X. H. \& Dong, X. P. Antimicrobial properties of berberines alkaloids in Coptis chinensis Franch by microcalorimetry. J. Biochem. Biophys. Methods 70, 845-849 (2008).

56. Flower, A., Wang, L. Q., Lewith, G., Liu, J. P. \& Li, Q. Chinese herbal medicine for treating recurrent urinary tract infections in women. Cochrane Database Syst Rev. 6, CD01044 (2015)

57. Ma, X. Y., Zhi, Y., Zhang, X., Zhao, H. \& Gao, G. J. Clinical study of Xianqing Houbu method in treating senile female recurrent urinary tract infection. J. Emergency Tradit. Chinese Med. 20, 1918-1919 (2011)

58. Shen, Y. \& Yao, Q. Clinical observation of "baitouweng decoction" and "erxian decoction" in treating lower urinary infection in 52 postmenopausal women. Shanghai J. Tradit. Chinese Med. 41, 37-38 (2007)

59. Zhao, K. S. et al. Clinical study of clearing liver fire removing dampness, strengthening spleen and tonifying kidney methods in treating middle-aged and old woman with chronic urinary tract infection. Int. J. Tradit. Chinese Med. 33, 976-978 (2011).

60. Chen, M., Wang, Y. \& Gu, X. C. Clinical observation of modified "erxian decoction" plus antibiotics in treating chronic urinary tract infection in middle-aged and old women. Shanghai J. Tradit. Chinese Med. 42, 48-49 (2008).

61. Luo, M. Clinical study of bushen tonglin decoction on female with chronic urinary tract infection. Hubei Uni. Chinese Med. (2011).

62. Gu, X. C., Xu, Z., Chen, M. \& Wang, M. Study of erding erxian docoction compared with sanjin tablet in treating recurrent urinary tract infection. Chinese J. Integr. Tradit. Western Nephrol. 12, 623-624 (2011)

63. Qin, S. G. Clinical analysis of Ningmitai capsule on 60 cases chronic urinary tract infection. Hebei Med. 10, 700-702 (2004).

64. Balshem, $\mathrm{H}$. et al. GRADE guidelines: 3 . Rating the quality of evidence. J. Clin. Epidemiol. 64, 401-406 (2011).

65. Guay, D. R. Cranberry and urinary tract infections. Drugs 69, 775-807 (2009).

66. Borukh, I. F., Kirbaba, V. I. \& Senchuk, G. V. [Antimicrobial properties of cranberry]. Voprosy Pitaniia 31, 82 (1972)

67. Hisano, M., Bruschini, H., Nicodemo, A. C. \& Srougi, M. Cranberries and lower urinary tract infection prevention. Clinics 67, 661-668 (2012)

68. Lynch, D. M. Cranberry for prevention of urinary tract infections. Am. Fam. Physician 70, 2175-2177 (2004).

69. Beachey, E. H. Bacterial adherence: adhesin-receptor interactions mediating the attachment of bacteria to mucosal surface. J. Infect. Dis. 143, 325-345 (1981)

70. Howell, A. B. Bioactive compounds in cranberries and their role in prevention of urinary tract infections. Mol. Nutr. Food Res. 51, 732-737 (2007).

71. Ofek, I. \& Beachey, E. H. Mannose binding and epithelial cell adherence of Escherichia coli. Infect. Immun. 22, 247-254 (1978).

72. Zafriri, D., Ofek, I., Adar, R., Pocino, M. \& Sharon, N. Inhibitory activity of cranberry juice on adherence of type 1 and type P fimbriated Escherichia coli to eucaryotic cells. Antimicrob. Agents Chemother. 33 92-98 (1989)

73. Hidalgo, G., Chan, M. \& Tufenkji, N. Inhibition of Escherichia coli CFT073 fliC expression and motility by cranberry materials. Appl. Environ. Microbiol. 77 6852-6857 (2011).

74. O'May, C. \& Tufenkji, N. The swarming motility of Pseudomonas aeruginosa is blocked by cranberry proanthocyanidins and other tannin-containing materials. Appl. Environ. Microbiol. 77, 3061-3067 (2011)

75. Howell, A. B. et al. A-Type cranberry proanthocyanidins and uropathogenic bacterial anti-adhesion activity. Phytochemistry 66, 2281-2291 (2005)

76. Liu, Y., Black, M. A., Caron, L. \& Camesano, T. A. Role of cranberry juice on molecular-scale surface characteristics and adhesion behavior of Escherichia coli. Biotechnol. Bioengineer. 93 297-305 (2006)

77. Vasileiou, I., Katsargyris, A., Theocharis, S. \& Giaginis, C. Current clinical status on the preventive effects of cranberry consumption against urinary tract infections. Nutr. Res. 33, 595-607 (2013)

78. Ren, D. et al. Differential gene expression for investigation of Escherichia coli biofilm inhibition by plant extract ursolic acid. Appl. Environ. Microbiol. 71, 4022-4034 (2005)

79. Grace, M. H., Massey, A. R., Mbeunkui, F., Yousef, G. C. \& Lila, M. A. Comparison of health-relevant flavonoids in commonly consumed cranberry products. J. Food Sci. 77, H176-H183 (2012).

80. Howell, A. B. \& Foxman, B. Cranberry juice and adhesion of antibiotic-resistant uropathogens. JAMA 287, 3082-3083 (2002).

81. Avorn, J. et al. Reduction of bacteriuria and pyuria after ingestion of cranberry juice. JAMA $\mathbf{2 7 1}$ 751-754 (1994).

82. Howell, A. B. et al. Dosage effect on uropathogenic Escherichia coli anti-adhesion activity in urine following consumption of cranberry powder standardized for proanthocyanidin content: a multicentric randomized double blind study. BMC Infect. Dis. 10, 94 (2010).

83. Beerepoot, M. \& Geerlings, S. Non-antibiotic prophylaxis for urinary tract infections. Pathogens $\mathbf{5}$, E36 (2016).

84. Luis, A., Domingues, F. \& Pereira, L. Can cranberries contribute to reduce the incidence of urinary tract infections? A systematic review with meta-analysis and trial sequential analysis of clinical trials. J. Urol. 198, 614-621 (2017)

85. Jepson, R. G., Williams, G. \& Craig J. C. Cranberries for preventing urinary tract infections. Cochrane Database Syst. Rev. 10, CD001321 (2012).

86. Wang, C. H. et al. Cranberry-containing products for prevention of urinary tract infections in susceptible populations: a systematic review and meta-analysis of randomized controlled trials. Arch. Intern. Med 172 988-996 (2012)

87. Stothers, L. A randomized trial to evaluate effectiveness and cost effectiveness of naturopathic cranberry products as prophylaxis against urinary tract infection in women Can J. Urol 9, 1558-1562 (2002).

88. Vostalova, J. et al. Are high proanthocyanidins key to cranberry efficacy in the prevention of recurrent urinary tract infection? Phytother. Res. 29, 1559-1567 (2015).

89. Maki, K. C. et al. Consumption of a cranberry juice beverage lowered the number of clinical urinary tract infection episodes in women with a recent history of urinary tract infection. Am. J. Clin. Nutr. 103 1434-1442 (2016).

90. Kontiokari, T. et al. Randomised trial of cranberrylingonberry juice and Lactobacillus GG drink for the prevention of urinary tract infections in women. BMJ 322, 1571 (2001)

91. Stapleton, A. E. et al. Recurrent urinary tract infection and urinary Escherichia coli in women ingesting cranberry juice daily: a randomized controlled trial. Mayo Clin. Proc. 87, 143-150 (2012).

92. Barbosa-Cesnik, C. et al. Cranberry juice fails to prevent recurrent urinary tract infection: results from a randomized placebo-controlled trial. Clin. Infect. Dis. 52, 23-30 (2011).

93. Foxman, B. et al. Risk factors for second urinary tract infection among college women. Am. J. Epidemiol. 151, 1194-1205 (2000)

94. McMurdo, M. E., Argo, I., Phillips, G., Daly, F. \& Davey, P. Cranberry or trimethoprim for the prevention of recurrent urinary tract infections? A randomized controlled trial in older women. J. Antimicrob. Chemother. 63, 389-395 (2009).

95. Beerepoot, M. A. et al. Cranberries versus antibiotics to prevent urinary tract infections: a randomized double-blind noninferiority trial in premenopausal women. Arch. Intern. Med. 171, 1270-1278 (2011).

96. Beerepoot, M. A., Geerlings, S. E., van Haarst, E. P. van Charante, N. M. \& ter Riet, G. Nonantibiotic prophylaxis for recurrent urinary tract infections: a systematic review and meta-analysis of randomized controlled trials. J. Urol. 190, 1981-1989 (2013).

97. Caljouw, M. A. et al. Effectiveness of cranberry capsules to prevent urinary tract infections in vulnerable older persons: a double-blind randomized placebo-controlled trial in long-term care facilities. J. Am. Geriatr. Soc. 62, 103-110 (2014).

98. Juthani-Mehta, M. et al. Effect of cranberry capsules on bacteriuria plus pyuria among older women in nursing homes: a randomized clinical trial. JAMA 316 , 1879-1887 (2016).

99. Afshar, K., Stothers, L., Scott, H. \& MacNeily, A. E. Cranberry juice for the prevention of pediatric urinary tract infection: a randomized controlled trial. J. Urol. 188, 1584-1587 (2012)

100. Ferrara, P. et al. Cranberry juice for the prevention of recurrent urinary tract infections: a randomized controlled trial in children. Scand. J. Urol. Nephrol. 43, 369-372 (2009).
101. Wing, D. A., Rumney, P. J., Preslicka, C. W. \& Chung, J. H. Daily cranberry juice for the prevention of asymptomatic bacteriuria in pregnancy: a randomized, controlled pilot study. J. Urol. 180 1367-1372 (2008).

102. Dugoua, J. J., Seely, D., Perri, D., Mills, E. \& Koren, G. Safety and efficacy of cranberry (Vaccinium macrocarpon) during pregnancy and lactation. Can. J. Clin. Pharmacol. 15, e80-e86 (2008).

103. Hannan, T. J., Mysorekar, I. U., Hung, C. S Isaacson-Schmid, M. L. \& Hultgren, S. J. Early severe inflammatory responses to uropathogenic $E$. coli predispose to chronic and recurrent urinary tract infection. PLoS Pathog. 6, e1001042 (2010)

104. Schlager, T. A., LeGallo, R., Innes, D., Hendley, J. O. $\&$ Peters, C. A. B cell infiltration and lymphonodular hyperplasia in bladder submucosa of patients with persistent bacteriuria and recurrent urinary tract infections. J. Urol. 186, 2359-2364 (2011).

105. Hannan, T. J. et al. Inhibition of cyclooxygenase-2 prevents chronic and recurrent cystitis. EBioMedicine 1, 46-57 (2014)

106. Wheeler, M. A., Hausladen, D. A., Yoon, J. H. $\&$ Weiss, R. M. Prostaglandin E2 production and cyclooxygenase-2 induction in human urinary tract infections and bladder cancer. J. Urol. 168, 1568-1573 (2002).

107. Bleidorn, J., Gagyor, I., Kochen, M. M., Wegscheider, K \& Hummers-Pradier, E. Symptomatic treatment (ibuprofen) or antibiotics (ciprofloxacin) for uncomplicated urinary tract infection?-results of a randomized controlled pilot trial. BMC Med. 8, 30 (2010).

108. Gagyor, I. et al. Ibuprofen versus fosfomycin for uncomplicated urinary tract infection in women: randomised controlled trial. BMJ 351, h6544 (2015).

109. Ferry, S. A., Holm, S. E., Stenlund, H., Lundholm, R. $\&$ Monsen, T. J. The natural course of uncomplicated lower urinary tract infection in women illustrated by a randomized placebo controlled study. Scand. J. Infect. Dis. 36, 296-301 (2004)

110. Christiaens, T. C. et al. Randomised controlled trial of nitrofurantoin versus placebo in the treatment of uncomplicated urinary tract infection in adult women. Br. J. Gen. Pract. 52, 729-734 (2002)

111. Kalle, A. M. \& Rizvi, A. Inhibition of bacterial multidrug resistance by celecoxib, a cyclooxygenase-2 inhibitor. Antimicrob. Agents Chemother. 55 439-442 (2011)

112. Velraeds, M. M., van der Mei, H. C., Reid, G. \& Busscher, H. J. Inhibition of initial adhesion of uropathogenic Enterococcus faecalis by biosurfactants from Lactobacillus isolates. Appl. Environ. Microbiol. 62, 1958-1963 (1996).

113. Bruce, A. W., Chadwick, P., Hassan, A. \& VanCott, G. F Recurrent urethritis in women. Can. Med. Assoc. J. 108, 973-976 (1973).

114. Reid, G., Bruce, A. W., Cook, R. L. \& Llano, M. Effect on urogenital flora of antibiotic therapy for urinary tract infection. Scand. J. Infect. Dis. 22 43-47 (1990)

115. Andreu, A., Stapleton, A. E., Fennell, C. L., Hillier, S. L. \& Stamm, W. E. Hemagglutination, adherence, and surface properties of vaginal Lactobacillus species. J. Infect. Dis. 171, 1237-1243 (1995).

116. Osset, J., Bartolome, R. M., Garcia, E. \& Andreu, A. Assessment of the capacity of Lactobacillus to inhibit the growth of uropathogens and block their adhesion to vaginal epithelial cells. J. Infect. Dis. 183, 485-491 (2001).

117. Mastromarino, P. et al. Characterization and selection of vaginal Lactobacillus strains for the preparation of vaginal tablets. J. Appl. Microbiol. 93, 884-893 (2002).

118. Barrons, R. \& Tassone, D. Use of Lactobacillus probiotics for bacterial genitourinary infections in women: a review. Clin. Ther. 30, 453-468 (2008).

119. Zarate, G. \& Nader-Macias, M. E. Influence of probiotic vaginal lactobacilli on in vitro adhesion of urogenital pathogens to vaginal epithelial cells. Lett. Appl. Microbiol. 43, 174-180 (2006)

120. de Llano, D. G. et al. Strain-specific inhibition of the adherence of uropathogenic bacteria to bladder cells by probiotic Lactobacillus spp. Pathog. Dis. https:// doi.org/10.1093/femspd/ftx043 (2017).

121. Manzoor, A., Ul-Haq, I., Baig, S., Qazi, J. I. \& Seratlic, S. Efficacy of locally isolated lactic acid bacteria against antibiotic-resistant uropathogens. Jundishapur J. Microbiol. 9, e18952 (2016).

122. Shim, Y. H., Lee, S. J. \& Lee, J. W. Antimicrobia activity of lactobacillus strains against uropathogens. Pediatr. Int. 58, 1009-1013 (2016). 
123. Aroutcheva, A. et al. Defense factors of vaginal lactobacilli. Am. J. Obstetr. Gynecol. 185, 375-379 (2001).

124. Atassi, F. \& Servin, A. L. Individual and co-operative roles of lactic acid and hydrogen peroxide in the killing activity of enteric strain Lactobacillus johnsonii NCC933 and vaginal strain Lactobacillus gasseri KS120.1 against enteric, uropathogenic and vaginosis-associated pathogens. FEMS Microbiol. Lett. 304, 29-38 (2010)

125. Alakomi, H. L. et al. Lactic acid permeabilizes gram negative bacteria by disrupting the outer membrane Appl. Environ. Microbiol. 66, 2001-2005 (2000)

126. Amdekar, S., Singh, V. \& Singh, D. D. Probiotic therapy: immunomodulating approach toward urinary tract infection. Curr. Microbiol. 63, 484-490 (2011).

127. Karlsson, M., Scherbak, N., Khalaf, H., Olsson, P. E. $\&$ Jass, J. Substances released from probiotic Lactobacillus rhamnosus GR-1 potentiate NF-кB activity in Escherichia coli-stimulated urinary bladder cells. FEMS Immunol. Med. Microbiol. 66, 147-156 (2012).

128. Fettweis, J. M. et al. Differences in vaginal microbiome in African American women versus women of European ancestry. Microbiology 160, 2272-2282 (2014).

129. Baerheim, A., Larsen, E. \& Digranes, A. Vaginal application of lactobacilli in the prophylaxis of recurrent lower urinary tract infection in women. Scand. J. Prim. Health Care 12, 239-243 (1994).

130. Colodner, R., Edelstein, H., Chazan, B. \& Raz, R. Vaginal colonization by orally administered Lactobacillus rhamnosus GG. Isr. Med. Assoc. J. 5 , 767-769 (2003)

131. Reid, G., Millsap, K. \& Bruce, A. W. Implantation of Lactobacillus casei var rhamnosus into vagina. Lancet 344, 1229 (1994).

132. Morelli, L., Zonenenschain, D., Del Piano, M. $\&$ Cognein, P. Utilization of the intestinal tract as a delivery system for urogenital probiotics. J. Clin. Gastroenterol. 38, S107-S110 (2004).

133. Cribby, S., Taylor, M. \& Reid, G. Vaginal microbiota and the use of probiotics. Interdiscip. Perspect. Infect. Dis. 2008, 256490 (2008).

134. Mezzasalma, V. et al. Orally administered multispecies probiotic formulations to prevent uro-genital infections: a randomized placebo-controlled pilot study. Arch Gynecol. Obstetr. 295, 163-172 (2017).

135. Reid, G. et al. Oral use of Lactobacillus rhamnosus GR-1 and L. fermentum RC-14 significantly alters vaginal flora: randomized, placebo-controlled trial in 64 healthy women. FEMS Immunol. Med. Microbiol. 35, 131-134 (2003)

136. Beerepoot, M. A. et al. Lactobacilli vs antibiotics to prevent urinary tract infections: a randomized, double blind, noninferiority trial in postmenopausal women Arch. Intern. Med. 172, 704-712 (2012).

137. Lee, S. J., Shim, Y. H., Cho, S. J. \& Lee, J. W. Probiotics prophylaxis in children with persistent primary vesicoureteral reflux. Pediatr. Nephrol. 22, 1315-1320 (2007)

138. Mohseni, M. J. et al. Combination of probiotics and antibiotics in the prevention of recurrent urinary tract infection in children. Iranian J. Pediatr. 23, 430-438 (2013)

139. Reid, G., Bruce, A. W. \& Taylor, M. Influence of three-day antimicrobial therapy and lactobacillus vaginal suppositories on recurrence of urinary tract infections. Clin. Ther. 14, 11-16 (1992).

140. Stapleton, A. E. et al. Randomized, placebo-controlled phase 2 trial of a Lactobacillus crispatus probiotic given intravaginally for prevention of recurrent urinary tract infection. Clin. Infect. Dis. 52, 1212-1217 (2011)

141. Schwenger, E. M., Tejani, A. M. \& Loewen, P. S. Probiotics for preventing urinary tract infections in adults and children. Cochrane Database Syst. Rev. 12 CD008772 (2015).

142. Grin, P. M., Kowalewska, P. M., Alhazzan, W. \& Fox-Robichaud, A. E. Lactobacillus for preventing recurrent urinary tract infections in women: metaanalysis. Can. J. Urol. 20, 6607-6614 (2013).

143. Davis, J. A. \& Freeze, H. H. Studies of mannose metabolism and effects of long-term mannose ingestion in the mouse. Biochim. Biophys. Acta 1528 116-126 (2001)

144. Fronzes, R., Remaut, H. \& Waksman, G. Architectures and biogenesis of non-flagellar protein appendages in Gram-negative bacteria. EMBO J. 27, 2271-2280 (2008).

145. Choudhury, D. et al. X-Ray structure of the FimC-FimH chaperone-adhesin complex from uropathogenic Escherichia coli. Science 285, 1061-1066 (1999).
146. Zhou, G. et al. Uroplakin la is the urothelial receptor for uropathogenic Escherichia coli: evidence from in vitro FimH binding. J. Cell Sci. 114, 4095-4103 (2001).

147. Pak, J., Pu, Y., Zhang, Z. T., Hasty, D. L. \& Wu, X. R. Tamm-Horsfall protein binds to type 1 fimbriated Escherichia coli and prevents E. coli from binding to uroplakin la and Ib receptors. J. Biol. Chem. 276, 9924-9930 (2001).

148. Eto, D. S., Jones, T. A., Sundsbak, J. L. \& Mulvey, M. A Integrin-mediated host cell invasion by type 1-piliated uropathogenic Escherichia coli. PLoS Pathog. 3, e100 (2007).

149. Mydock-McGrane, L. K., Cusumano, Z. T. $\delta$ Janetka, J. W. Mannose-derived FimH antagonists: a promising anti-virulence therapeutic strategy for urinary tract infections and Crohn's disease. Expert Opin. Ther. Patents 26, 175-197 (2016).

150. Michaels, E. K., Chmiel, J. S., Plotkin, B. J. \& Schaeffer, A. J. Effect of D-mannose and D-glucose on Escherichia coli bacteriuria in rats. Urol. Res. 11 97-102 (1983).

151. Schaeffer, A. J., Chmiel, J. S., Duncan, J. L. \& Falkowski, W. S. Mannose-sensitive adherence of Escherichia coli to epithelial cells from women with recurrent urinary tract infections. J. Urol. 131, 906-910 (1984)

152. Wellens, A. et al. Intervening with urinary tract infections using anti-adhesives based on the crystal structure of the FimH-oligomannose-3 complex. PLOS ONE 3, e2040 (2008).

153. Cusumano, C. K. et al. Treatment and prevention of urinary tract infection with orally active FimH inhibitors. Sci. Transl Med. 3, 109ra115 (2011).

154. Spaulding, C. N. et al. Selective depletion of uropathogenic $E$. coli from the gut by a FimH antagonist. Nature 546, 528-532 (2017).

155. Mydock-McGrane, L. K., Hannan, T. J. \& Janetka, J. W. Rational design strategies for FimH antagonists: new drugs on the horizon for urinary tract infection and Crohn's disease. Expert Opin. Drug Discov. 12 711-731 (2017)

156. Kranjcec, B., Papes, D. \& Altarac, S. D-Mannose powder for prophylaxis of recurrent urinary tract infections in women: a randomized clinical trial. World J. Urol. 32, 79-84 (2014).

157. Phe, V. et al. Open label feasibility study evaluating D-mannose combined with home-based monitoring of suspected urinary tract infections in patients with multiple sclerosis. Neurourol. Urodyn. 36 1770-1775 (2017).

158. Vicariotto, F. Effectiveness of an association of a cranberry dry extract, D-mannose, and the two microorganisms Lactobacillus plantarum LP01 and Lactobacillus paracasei LPC09 in women affected by cystitis: a pilot study. J. Clin. Gastroenterol. 48 , S96-S101 (2014).

159. Naves, P. et al. Effects of human serum albumin, ibuprofen and $\mathrm{N}$-acetyl-L-cysteine against biofilm formation by pathogenic Escherichia coli strains. J. Hosp. Infect. 76, 165-170 (2010).

160. Palleschi, G. et al. Prospective study to compare antibiosis versus the association of $\mathrm{N}$-acetylcysteine D-mannose and Morinda citrifolia fruit extract in preventing urinary tract infections in patients submitted to urodynamic investigation. Arch. Ital. Urol. Androl. 89, 45-50 (2017).

161. Hamilton-Miller, J. M. \& Brumfitt, W. Methenamine and its salts as urinary tract antiseptics: variables affecting the antibacterial activity of formaldehyde, mandelic acid, and hippuric acid in vitro. Invest. Urol. 14, 287-291 (1977).

162. Neely, W. B. Action of formaldehyde on microorganisms. III. Bactericidal action of sublethal concentrations of formaldehyde on aerobacter aerogenes. J. Bacteriol. 86, 445-448 (1963)

163. Musher, D. M. \& Griffith, D. P. Generation of formaldehyde from methenamine: effect of $\mathrm{pH}$ and concentration, and antibacterial effect. Antimicrob. Agents Chemother. 6, 708-711 (1974).

164. Lee, B. S., Bhuta, T., Simpson, J. M. \& Craig, J. C. Methenamine hippurate for preventing urinary tract infections. Cochrane Database Syst. Rev. 10 CD003265 (2012)

165. Nahata, M. C., Cummins, B. A., McLeod, D. C. \& Butler, R. Predictability of methenamine efficacy based on type of urinary pathogen and pH. J. Am. Geriatr. Soc. 29, 236-239 (1981)

166. Nahata, M. C., Cummins, B. A., McLeod, D. C., Schondelmeyer, S. W. \& Butler, R. Effect of urinary acidifiers on formaldehyde concentration and efficacy with methenamine therapy. Eur. J. Clin. Pharmacol. 22, 281-284 (1982)

167. Sander, S. \& Jakobsen, A. Jr. [Preventive Hiprex in urinary tract operations]. Tidsskr. Nor. Laegeforen. 96, 167-169 (1976)

168. Thomlinson, J., Williams, J. D. \& Cope, E. Persistence of bacteriuria following gynaecological surgery: a trial of methenamine hippurate. Br. J. Urol. 40, 479-482 (1968).

169. ISRCTN registry. Alternatives to prophylactic antibiotics for the treatment of recurrent urinary tract infection in women. BMC http://www.isrctn.com/ ISRCTN70219762 (2016).

170. Mulvey, M. A., Schilling, J. D., Martinez, J. J. \& Hultgren, S. J. Bad bugs and beleaguered bladders: interplay between uropathogenic Escherichia coli and innate host defenses. Proc. Natl Acad. Sci. USA 97. 8829-8835 (2000).

171. Teng, J., Wang, Z. Y., Jarrard, D. F. \& Bjorling, D. E. Roles of estrogen receptor $\alpha$ and $\beta$ in modulating urothelial cell proliferation. Endocr. Relat. Cancer 15 351-364 (2008).

172. Simpson, E. R. Sources of estrogen and their importance. J. Steroid Biochem. Mol. Biol. 86, 225-230 (2003).

173. Raz, R. Hormone replacement therapy or prophylaxis in post-menopausal women with urinary tract infection. J. Infect. Dis. 183, 74-76 (2001).

174. Marshburn, P. B. \& Carr, B. R. Hormone replacement therapy. Protection against the consequences of menopause. Postgrad. Med. 92, 145-148, 151-142, 157-149 (1992).

175. Luthje, P., Hirschberg, A. L. \& Brauner, A. Estrogenic action on innate defense mechanisms in the urinary tract. Maturitas 77, 32-36 (2014)

176. Robinson, D. \& Cardozo, L. Oestrogens and the lower urinary tract. BJOG 111 (Suppl. 1), 10-14 (2004).

177. Hannan, T. J., Hooton, T. M. \& Hultgren, S. J. Estrogen and recurrent UTI: what are the facts? Sci. Transl Med. 5, $190 \mathrm{fs} 123$ (2013).

178. Mirmonsef, P. et al. Exploratory comparison of vaginal glycogen and Lactobacillus levels in premenopausal and postmenopausal women. Menopause 22, 702-709 (2015)

179. Miller, L. et al. Depomedroxyprogesterone-induced hypoestrogenism and changes in vaginal flora and epithelium. Obstetr. Gynecol. 96, 431-439 (2000)

180. Raz, R. \& Stamm, W. E. A controlled trial of intravaginal estriol in postmenopausal women with recurrent urinary tract infections. N. Engl. J. Med. 329, 753-756 (1993)

181. Chromek, M. et al. The antimicrobial peptide cathelicidin protects the urinary tract against invasive bacterial infection. Nat. Med. 12, 636-641 (2006).

182. Luthje, P. et al. Estrogen supports urothelial defense mechanisms. Sci. Transl Med. 5, 190ra180 (2013).

183. Stern, J. A., Hsieh, Y. C. \& Schaeffer, A. J. Residual urine in an elderly female population: novel implications for oral estrogen replacement and impact on recurrent urinary tract infection. J. Urol. 171, 768-770 (2004).

184. Christiansen, C. \& Riis, B. J. $17 \beta$-estradiol and continuous norethisterone: a unique treatment for established osteoporosis in elderly women. J. Clin. Endocrinol. Metab. 71, 836-841 (1990).

185. Rossouw, J. E. et al. Postmenopausal hormone therapy and risk of cardiovascular disease by age and years since menopause. JAMA 297, 1465-1477 (2007).

186. Cardozo, L., Lose, G., McClish, D. \& Versi, E. A systematic review of the effects of estrogens for symptoms suggestive of overactive bladder. Acta Obstet. Gynecol. Scand. 83, 892-897 (2004).

187. Brown, J. S. et al. Urinary tract infections in postmenopausal women: effect of hormone therapy and risk factors. Obstetr. Gynecol. 98, 1045-1052 (2001).

188. Cardozo, L., Benness, C. \& Abbott, D. Low dose oestrogen prophylaxis for recurrent urinary tract infections in elderly women. Br. J. Obstetr. Gynaecol. 105, 403-407 (1998).

189. Kirkengen, A. L. et al. Oestriol in the prophylactic treatment of recurrent urinary tract infections in postmenopausal women. Scand. J. Prim. Health Care 10, 139-142 (1992)

190. Ouslander, J. G. et al. Effects of oral estrogen and progestin on the lower urinary tract among female nursing home residents. J. Am. Geriatr. Soc. 49 , 803-807 (2001)

191. Perrotta, C., Aznar, M., Mejia, R., Albert, X. $\& \mathrm{Ng}, \mathrm{C}$. W. Oestrogens for preventing recurrent 
urinary tract infection in postmenopausal women. Cochrane Database Syst. Rev. 2, CD005131 (2008).

192. Raz, R. et al. Effectiveness of estriol-containing vaginal pessaries and nitrofurantoin macrocrystal therapy in the prevention of recurrent urinary tract infection in postmenopausal women. Clin. Infect. Dis. 36, 1362-1368 (2003)

193. Suckling, J., Lethaby, A. \& Kennedy, R. Local oestrogen for vaginal atrophy in postmenopausal women. Cochrane Database Syst. Rev. 4, CD001500 (2006).

194. Eriksen, B. A randomized, open, parallel-group study on the preventive effect of an estradiol-releasing vaginal ring (Estring) on recurrent urinary tract infections in postmenopausal women. Am. J. Obstetr. Gynecol. 180, 1072-1079 (1999).

195. Xu, R., Wu, Y. \& Hu, Y. [Prevention and treatment of recurrent urinary system infection with estrogen cream in postmenopausal women]. Zhonghua Fu Chan Ke Za Zhi 36, 531-533 (2001).

196. Parsons, C. L., Boychuk, D., Jones, S., Hurst, R. \& Callahan, H. Bladder surface glycosaminoglycans: an epithelial permeability barrier. J. Urol. 143, 139-142 (1990).

197. Parsons, C. L., Pollen, J. J., Anwar, H., Stauffer, C. \& Schmidt, J. D. Antibacterial activity of bladder surface mucin duplicated in the rabbit bladder by exogenous glycosaminoglycan (sodium pentosanpolysulfate). Infect. Immun. 27, 876-881 (1980).

198. Parsons, C. L., Greenspan, C. \& Mulholland, S. G. The primary antibacterial defense mechanism of the bladder. Invest. Urol. 13, 72-78 (1975).

199. Ruggieri, M. R., Hanno, P. M. \& Levin, R. M. The effects of heparin on the adherence of five species of urinary tract pathogens to urinary bladder mucosa. Urol. Res. 12, 199-203 (1984).

200. Parsons, C. L. The role of the urinary epithelium in the pathogenesis of interstitial cystitis/prostatitis/urethritis Urology 69, 9-16 (2007).

201. Cicione, A. et al. Restoring the glycosaminoglycans layer in recurrent cystitis: experimental and clinical foundations. Int. J. Urol. 21, 763-768 (2014).

202. Lee, D. G. et al. Preventive effects of hyaluronic acid on Escherichia coli-induced urinary tract infection in rat. Urology 75, 949-954 (2010).

203. Hauser, P. J. et al. Restoring barrier function to acid damaged bladder by intravesical chondroitin sulfate J. Urol. 182, 2477-2482 (2009).

204. Yildiz, N. et al. Intravesical hyaluronic acid treatment improves bacterial cystitis and reduces cystitis-induced hypercontractility in rats. Int. J. Urol. 22, 598-603 (2015).

205. Tasdemir, S. et al. Intravesical hyaluronic acid and chondroitin sulfate alone and in combination fo urinary tract infection: assessment of protective effects in a rat model. Int. J. Urol. 19, 1108-1112 (2012).

206. Nishimura, M. et al. Role of chondroitin sulfatehyaluronan interactions in the viscoelastic properties of extracellular matrices and fluids. Biochim. Biophys. Acta 1380, 1-9 (1998)

207. Freissler, E., Meyer auf der Heyde, A., David, G. Meyer, T. F. \& Dehio, C. Syndecan-1 and syndecan-4 can mediate the invasion of OpaHSPG-expressing Neisseria gonorrhoeae into epithelial cells. Cell. Microbiol. 2, 69-82 (2000).

208. Laquerre, S. et al. Heparan sulfate proteoglycan binding by herpes simplex virus type 1 glycoproteins $\mathrm{B}$ and $\mathrm{C}$, which differ in their contributions to virus attachment, penetration, and cell-to-cell spread. J. Virol. 72, 6119-6130 (1998).

209. Constantinides, C. et al. Prevention of recurrent bacterial cystitis by intravesical administration of hyaluronic acid: a pilot study. BJU Int. 93, 1262-1266 (2004).

210. Lipovac, M. et al. Prevention of recurrent bacterial urinary tract infections by intravesical instillation of hyaluronic acid. Int. J. Gynaecol. Obstetr. 96, 192-195 (2007).

211. Damiano, R. et al. Prevention of recurrent urinary tract infections by intravesical administration of hyaluronic acid and chondroitin sulphate: a placebocontrolled randomised trial. Eur. Urol. 59, 645-651 (2011)

212. De Vita, D. \& Giordano, S. Effectiveness of intravesical hyaluronic acid/chondroitin sulfate in recurrent bacterial cystitis: a randomized study. Int. Urogynecol J. 23, 1707-1713 (2012)

213. Cicione, A. et al. Intravesical treatment with highlyconcentrated hyaluronic acid and chondroitin sulphate in patients with recurrent urinary tract infections: results from a multicentre survey. Can. Urol. Assoc. J. 8, E721-E727 (2014).

214. Gugliotta, G. et al. Is intravesical instillation of hyaluronic acid and chondroitin sulfate useful in preventing recurrent bacterial cystitis? A multicenter case control analysis. Taiwan J. Obstet. Gynecol. $\mathbf{5 4}$ 537-540 (2015)

215. Torella, M. et al. Intravesical therapy in recurrent cystitis: a multi-center experience. J. Infect. Chemother 19, 920-925 (2013)

216. Ciani, O. et al. Intravesical administration of combined hyaluronic acid (HA) and chondroitin sulfate (CS) for the treatment of female recurrent urinary tract infections: a European multicentre nested case-control study. BMJ Open 6, e009669 (2016).

217. De Vita, D., Antell, H. \& Giordano, S. Effectiveness of intravesical hyaluronic acid with or without chondroitin sulfate for recurrent bacterial cystitis in adult women: a meta-analysis. Int. Urogynecol J. 24, 545-552 (2013).

218. Chan, C. Y., St John, A. L. \& Abraham, S. N. Mast cell interleukin-10 drives localized tolerance in chronic bladder infection. Immunity 38, 349-359 (2013).

219. Brumbaugh, A. R. \& Mobley, H. L. Preventing urinary tract infection: progress toward an effective Escherichia coli vaccine. Expert Rev. Vaccines 11 663-676 (2012)

220. Schmidhammer, S. et al. An Escherichia coli-based oral vaccine against urinary tract infections potently activates human dendritic cells. Urology 60, 521-526 (2002).

221. Van Pham, T., Kreis, B., Corradin-Betz, S., Bauer, J. $\&$ Mauel, J. Metabolic and functional stimulation of lymphocytes and macrophages by an Escherichia coli extract (OM-89): in vitro studies. J. Biol. Response Mod. 9, 231-240 (1990)

222. Ha, U. S. \& Cho, Y. H. Immunostimulation with Escherichia coli extract: prevention of recurrent urinary tract infections. Int. J. Antimicrob. Agents 31 , S63-S67 (2008)

223. Huber, M., Baier, W., Serr, A. \& Bessler, W. G Immunogenicity of an $E$. coli extract after oral or intraperitoneal administration: induction of antibodies against pathogenic bacterial strains. Int J. Immunopharmacol. 22, 57-68 (2000).

224. Lee, S. J., Kim, S. W., Cho, Y. H. \& Yoon, M. S Anti-inflammatory effect of an Escherichia coli extract in a mouse model of lipopolysaccharideinduced cystitis. World J. Urol. 24, 33-38 (2006).

225. Wagenlehner, F. M. et al. A randomized, double-blind parallel-group, multicenter clinical study of Escherichia coli-lyophilized lysate for the prophylaxis of recurrent uncomplicated urinary tract infections. Urol. Intern. 95, 167-176 (2015)

226. Naber, K. G., Cho, Y. H., Matsumoto, T. \& Schaeffer, A. J. Immunoactive prophylaxis of recurrent urinary tract infections: a meta-analysis. Int. J. Antimicrob. Agents 33, 111-119 (2009).

227. Bonkat, G. et al. European Association of Urology Guidelines on urological infections. EAU https://uroweb.org/guideline/urological-infections/ (2018).

228. Das, P. Vaginal vaccine for recurrent urinary-tract infections. Lancet Infect. Dis. 2, 68 (2002).

229. Hopkins, W. J., Elkahwaji, J., Beierle, L. M. Leverson, G. E. \& Uehling, D. T. Vaginal mucosal vaccine for recurrent urinary tract infections in women: results of a phase 2 clinical trial. J. Urol. 177 , 1349-1353 (2007)

230. Uehling, D. T., Hopkins, W. J., Elkahwaji, J. E., Schmidt, D. M. \& Leverson, G. E. Phase 2 clinical trial of a vaginal mucosal vaccine for urinary tract infections. J. Urol. 170, 867-869 (2003).

231. Benito-Villalvilla, C. et al. MV140, a sublingual polyvalent bacterial preparation to treat recurren urinary tract infections, licenses human dendritic cells for generating Th 1, Th 17, and IL-10 responses via Syk and MyD88. Mucosal Immunol. 10, 924-935 (2017).

232. Lorenzo-Gomez, M. F. et al. Comparison of sublingual therapeutic vaccine with antibiotics for the prophylaxis of recurrent urinary tract infections. Frontiers Cell. Infect. Microbiol. 5, 50 (2015).

233. Yang, B. \& Foley, S. First experience in the UK of treating women with recurrent urinary tract infections with the bacterial vaccine Uromune ${ }^{\oplus}$. BJU Int. 121, 289-292 (2018)
234. Lorenzo-Gomez, M. F. et al. Evaluation of a therapeutic vaccine for the prevention of recurrent urinary tract infections versus prophylactic treatment with antibiotics. Int. Urogynecol J. 24, 127-134 (2013).

235. US National Library of Medicine. ClinicalTrials.gov https://clinicaltrials.gov/ct2/show/NCT02543827. (2018).

236. Marinova, S. et al. Cellular and humoral systemic and mucosal immune responses stimulated by an oral polybacterial immunomodulator in patients with chronic urinary tract infections. Int. J. Immunopathol. Pharmacol. 18, 457-473 (2005).

237. Langermann, S. et al. Vaccination with FimH adhesin protects cynomolgus monkeys from colonization and infection by uropathogenic Escherichia coli. J. Infect. Dis. 181, 774-778 (2000).

238. Dupuis, M. et al. Dendritic cells internalize vaccine adjuvant after intramuscular injection. Cell. Immunol. 186, 18-27 (1998).

239. Asadi Karam, M. R., Oloomi, M., Mahdavi, M., Habibi, M. \& Bouzari, S. Vaccination with recombinant FimH fused with flagellin enhances cellular and humoral immunity against urinary tract infection in mice. Vaccine 31, 1210-1216 (2013).

240. Roberts, J. A et al. Antibody responses and protection from pyelonephritis following vaccination with purified Escherichia coli PapDG protein. J. Urol. 171, 1682-1685 (2004)

241. Goluszko, P. et al. Vaccination with purified Dr Fimbriae reduces mortality associated with chronic urinary tract infection due to Escherichia coli bearing Dr adhesin. Infect. Immun. 73, 627-631 (2005).

242. Flores-Mireles, A. L., Pinkner, J. S., Caparon, M. G. \& Hultgren, S. J. EbpA vaccine antibodies block binding of Enterococcus faecalis to fibrinogen to prevent catheter-associated bladder infection in mice. Sci. Transl Med. 6, 254ra1 27 (2014).

243. Brumbaugh, A. R., Smith, S. N. \& Mobley, H. L. Immunization with the yersiniabactin receptor, FyuA, protects against pyelonephritis in a murine model of urinary tract infection. Infect. Immun. 81, 3309-3316 (2013).

244. van den Dobbelsteen, G. P. et al. Immunogenicity and safety of a tetravalent $E$. coli $\mathrm{O}$-antigen bioconjugate vaccine in animal models. Vaccine 34, 4152-4160 (2016).

245. Huttner, A. et al. Safety, immunogenicity, and preliminary clinical efficacy of a vaccine against extraintestinal pathogenic Escherichia coli in women with a history of recurrent urinary tract infection a randomised, single-blind, placebo-controlled phase $1 \mathrm{~b}$ trial. Lancet Infect. Dis. 17, 528-537 (2017).

246. Darouiche, R. O. et al. Multicenter randomized controlled trial of bacterial interference for prevention of urinary tract infection in patients with neurogenic bladder. Urology 78, 341-346 (2011).

247. Sunden, F., Hakansson, L., Ljunggren, E. \& Wullt, B. Escherichia coli 83972 bacteriuria protects against recurrent lower urinary tract infections in patients with incomplete bladder emptying. J. Urol. 184, 179-185 (2010).

248. Koves, B. et al. Rare emergence of symptoms during long-term asymptomatic Escherichia coli 83972 carriage without an altered virulence factor repertoire. J. Urol. 191, 519-528 (2014).

\section{Author contributions}

N.S., A.G., R.Z., A.S. and S.M. researched data for the article S.M. made substantial contributions to discussions of content. All authors wrote the manuscript, and S.M. reviewed and edited the manuscript before submission.

\section{Competing interests}

N.S., A.G. and R.Z declare no competing interests. A.S. has received an unrestricted educational grant is an adviser to and has received speaker fees from Allergan Ltd. and has received monies from Medtronic for promotional purposes. S.M. has received monies from Medtronic for promotional purposes.

\section{Publisher's note}

Springer Nature remains neutral with regard to jurisdictional claims in published maps and institutional affiliations.

\section{Reviewer information}

Nature Reviews Urology thanks T. J. Hannan, F. Wagenlehner and the other anonymous reviewer(s) for their contribution to the peer review of this work. 